\title{
Chemical diversification of simple synthetic antibodies
}

Mariha Islam, ${ }^{\dagger, \perp}$ Haixing P. Kehoe, ${ }^{\dagger, \perp}$ Jacob B. Lissoos, ${ }^{\dagger}$ Manjie Huang, ${ }^{\dagger}$ Christopher E. Ghadban, ${ }^{\dagger}$ Gregory I. Berumen,${ }^{\dagger}$ Hanan Z. Lane,${ }^{\dagger}$ and James A. Van Deventer ${ }^{\dagger, \downarrow, \star}$

${ }^{\dagger}$ Chemical and Biological Engineering Department, Tufts University, Medford, Massachusetts 02155 , United States

‡Biomedical Engineering Department, Tufts University, Medford, Massachusetts 02155, United States

${ }^{\perp}$ These authors contributed equally.

${ }^{*}$ Corresponding author

James.Van_Deventer@tufts.edu 


\section{Abstract}

Antibodies possess properties that make them valuable as therapeutics, diagnostics, and basic research tools. However, antibody chemical reactivity and covalent antigen binding are constrained, or even prevented, by the narrow range of chemistries encoded in the canonical amino acids. In this work, we investigate strategies for leveraging an expanded range of chemical functionality to augment antibody binding properties. Using yeast displayed antibodies, we explored the presentation of noncanonical amino acids (ncAAs) in or near antibody complementarity determining regions (CDRs) and evaluated the properties of the resulting constructs. To enable systematic characterization of ncAA incorporation sites, we first investigated whether diversification of a single antibody loop would support isolation of binding clones. We constructed a billion-member library containing canonical amino acid diversity and loop length diversity only within the 3rd complementarity determining region of the heavy chain (CDR-H3). Screens against a series of immunoglobulins from three species resulted in the isolation of antibodies exhibiting moderate affinities (double- to triple-digit nanomolar affinities) and, in several cases, single-species specificity. These findings confirmed that antibody specificity can be mediated by a single CDR. With this constrained diversity, we were able to utilize additional CDRs for the installation of chemically reactive and photo-crosslinkable ncAAs. Apparent binding affinities of ncAA-substituted synthetic antibodies on the yeast surface revealed that ncAA incorporation is generally well tolerated. However, changes in binding affinities did occur upon substitution, and varied based on factors including ncAA side chain identity, location of ncAA incorporation, and the ncAA incorporation machinery used. We further investigated chemical modifications facilitated by ncAA installation. Multiple azide-containing ncAAs supported both copper-catalyzed azide-alkyne cycloaddition (CuAAC) and strain-promoted azide-alkyne cycloaddition (SPAAC) without abrogation of binding function following the installation of bulky probes. Similarly, several alkyne substitutions facilitated CuAAC without apparent disruption of binding function. Finally, antibodies substituted with a photo-crosslinkable ncAA were evaluated for ultraviolet-mediated crosslinking on the yeast surface. Competition-based assays revealed position-dependent linkages that could not be displaced by excess soluble antigen, strongly suggesting successful crosslinking. Key findings regarding CuAAC reactions and photocrosslinking on the yeast surface were confirmed using soluble forms of ncAA-substituted clones. These consistent behaviors between the yeast surface and in solution suggest that chemical diversification can be incorporated into yeast display screening approaches. Taken together, our results highlight the power of integrating the use of yeast display and ncAAs in search of proteins with "chemically augmented" binding functions. More specifically, our findings provide the means to productively integrate antibodies with ncAAs by leveraging simple synthetic antibodies. The efficient preparation and chemical diversification of antibodies on the yeast surface opens up new possibilities for discovering "drug-like" protein leads in high throughput. 
Keywords: yeast display, synthetic antibodies, noncanonical amino acid, amber suppression, click chemistry, photo-crosslinking 


\section{Introduction}

Antibodies exhibit a versatile range of molecular recognition properties that make them cornerstones of therapeutics, diagnostics, and basic research tools. ${ }^{1-9}$ The rise of display technologies such as yeast and phage display has greatly expanded the range of methods for antibody discovery, characterization, and engineering. ${ }^{1,10-12}$ In addition to facilitating new approaches to mining antibody repertoires from immunologically derived sources, ${ }^{13-16}$ display technologies have led to the establishment of powerful, laboratory designed synthetic antibody libraries. ${ }^{17-21}$ Specifically, constraining antibody repertoires with synthetic approaches has led to important insights into how antibodies recognize target antigens. ${ }^{1,17-18,22-27}$ For example, researchers have found that restricting amino acid diversification to a single $\mathrm{CDR},{ }^{27}$ or limiting the diversification of antibody complementarity determining regions (CDRs) to as few as two amino acids $^{24-26,28}$ results in antibody repertoires capable of yielding antibodies with moderate target affinities (typically double-digit nanomolar to micromolar). Discoveries of functional antibodies from these studies and others indicate that antibody variable domains tolerate amino acid substitution patterns far beyond those employed by mammalian immune systems. These findings raise the following question: how extensively can antibody functions can be altered using chemical groups that are not genetically encoded in natural repertoires?

Several strategies for expanding the chemical repertoire of antibodies are feasible ${ }^{29-32}$ including posttranslational modification of antibody structures containing canonical amino acids ${ }^{31}$, 33-34 and the introduction of genetically encoded noncanonical amino acids (ncAAs). ${ }^{32,}{ }^{35-38}$ Numerous approaches to antibody chemical diversification have been explored extensively within the context of antibody-drug conjugates (ADCs) $\cdot{ }^{39-46}$ However, most ADC development strategies focus on modification sites located in antibody constant regions that have little or no effect on antigen binding; ${ }^{46-52}$ the result is modular addition of new chemistries that leave antibody binding function unchanged. In contrast, only sparse studies exist that examine the effects of adding 
chemical functionality near antibody complementarity determining regions (CDRs). Multiple groups have reported studies in which introducing ncAAs in antibody CDRs leads to altered antibody binding functions. Tirrell and coworkers observed that replacing the methionines of antidigoxin antibody variants with the azide-containing ncAA azidohomoalanine unexpectedly results in clones exhibiting increased digoxin binding affinity. ${ }^{53}$ Sakamoto and coworkers recently reported improved antigen affinities in two antibodies upon substituting halogenated tyrosines in place of tyrosines. ${ }^{38}$ Multiple groups have integrated ncAAs containing side chains known to interact with target antigens into antibodies for the purpose of phage display screening (Schultz, Smider, and coworkers) $)^{54-56}$ and systematic biochemical characterizations (Chang Liu and coworkers $)^{57}$ to identify antibody constructs with binding properties improved by the use of one or more ncAAs. Several strategies for altering antibody binding functions with an expanded chemical repertoire have used antibodies containing only canonical amino acids as starting points. Barbas, Lerner, and coworkers described the use of catalytic antibodies to present peptide binders within antibody variable domains, effectively replacing antibody CDRs with peptide-mediated antigen recognition. ${ }^{58-59}$ Multiple groups have leveraged thiol-mediated chemical conjugation strategies to present small molecules within antibody variable domains. For example, Winter and coworkers reported a phage display antibody library encoding a cysteine within CDR-L3 in order to introduce fluorescent dyes; library screening against a model antigen led to a clone exhibiting both dyedependent binding and changes in fluorescence properties upon antigen binding.$^{60}$ Finally, Wang, Miranda, and coworkers reported the design and construction of bivalent small molecule-antibody "hybrids" based on a crystal structure of a dipeptidyl peptidase IV (DPPIV)-antibody complex. ${ }^{61}$ Introduction of analogs of a known small molecule inhibitor of DPPIV into the noninhibitory antibody led to hybrid inhibitors with enhanced IC50 values in comparison to controls. Taken together, these studies indicate that there are several promising routes for diversifying the chemical groups that can be presented within antibody variable domains. Some of these studies also provide powerful examples of modulating antibody binding function with additional chemical 
groups. While promising, these studies also highlight opportunities to further generalize and expand approaches to the presentation of chemical groups within antibody structures.

In this work, we sought to establish strategies for generating simple synthetic antibodies and integrating chemical groups into the CDRs of the resulting clones. To facilitate efficient synthetic antibody discovery and derivatization, we utilized the combination of yeast display and noncanonical amino acid (ncAA) incorporation. ${ }^{62-64}$ We first developed an extremely simple, yeast-displayed synthetic single-chain variable fragment (scFv) library with amino acid and loop length diversity encoded solely in CDR-H3 ${ }^{27,65-66}$ Construction and screening of a billion-member yeast display library enabled the isolation of antibodies that exhibit specificity towards only one of three structurally similar antigens (donkey, bovine, or rabbit IgG). These findings demonstrate that even conventional amino acid diversity within a single CDR is sufficient to isolate specific antibodies on the yeast surface, in line with synthetic antibody libraries prepared in other display formats. ${ }^{25-28}$ Limiting library diversification, and presumably antigen binding in isolated clones, to CDR-H3 enabled us to incorporate ncAAs within additional CDRs of multiple clones and evaluate the effects of these insertions on scFv function. We observed retention of antigen binding after substitutions with multiple ncAAs containing azide or alkyne side chains at three different CDR substitution positions in several clones. However, we also noted changes in apparent binding affinities that depended on the substitution site, specific ncAA substitution utilized, and clone identity. For clones that retained binding function, all of the substitutions that we investigated supported copper-catalyzed azide-alkyne cycloadditions (CuAAC; azides and alkynes) and strainpromoted azide-alkyne cycloadditions (SPAAC; azides) "click" chemistries. Surprisingly, numerous ncAA-substituted clones supported efficient chemical reactions while also retaining binding function following modification, indicating that even reactions resulting in the introduction of large substituents can be accommodated in these clones. Finally, we evaluated whether clones containing the photo-crosslinkable ncAA p-azido-L-phenylalanine (AzF) would support crosslinking on the yeast surface. In two different clones, we observed covalent crosslinking with 
efficiencies varying as a function of the position of the ncAA substitution site. These key observations were further confirmed by conducting crosslinking experiments with soluble forms of one series of ncAA-substituted scFvs. Overall, our work demonstrates the feasibility of introducing ncAAs within several CDRs of simple synthetic antibodies, frequently without abrogating binding function. In addition, these simple, ncAA-substituted antibodies exhibit chemical reactivity and photoreactivity properties that cannot be accessed with canonical amino acids alone. Our findings expand the range of engineering strategies that can be used to present additional chemistries within antibodies (and other binding proteins), providing opportunities for tailoring and expanding the functions of these proteins for use in basic research and therapeutic discovery.

\section{Results and Discussion}

\section{Library Design, Construction and Characterization}

We first constructed an scFv library in yeast display format in which we employed a single antibody framework and introduced amino acid diversity within a single CDR (Figure 1). Similar to previously described synthetic antibody libraries, we utilized framework regions found in trastuzumab (Herceptin), which come from germlines known to tolerate a wide range of CDR diversification schemes. ${ }^{19,}$ 27, 67-68 The light chain was based on IGKV1-39 V domain and JK4 Kappa joining segment. The heavy chain was based on the IGHV3-23 V domain and JH4 joining segment. Amino acid sequences in the light chain CDRs (L1-L3) and heavy chain CDRs H1 and $\mathrm{H} 2$ are identical to those found in the immunogenetics database entries (http://www.imgt.org/) reporting the amino acid sequences of the $V$ domain and joining segment sequences (see also Materials and Methods). To leave the majority of the scFv available for exploring different sites for noncanonical amino acid incorporation, genetic diversity was limited to the CDR-H3 loop using the scheme depicted in Figure $1 \mathrm{~A}$. Amino acid diversity in CDR-H3 was designed to mimic the amino acid frequencies observed in natural human antibodies using degenerate codons with 
customized oligonucleotides containing the frequencies of nucleotide bases ${ }^{69}$ as shown in Figure 1A. Clones in the library contained a stretch of 5-13 amino acids encoded by "XYZ" codons as previously described, followed by one position encoding either A or $\mathrm{G}$ and one position encoding F/I/L/M for a total CDR-H3 loop length variation between 9 and 17 codons (Figure 1A; CDR-H3 ranges between positions $98-105$ in the Kabat numbering scheme used here ${ }^{70}$ ). This diversification scheme allowed 48 different possible sequences in each "XYZ" codon, two sequences within the $A / G$ codons, and six sequences within the F/I/L/M codons.
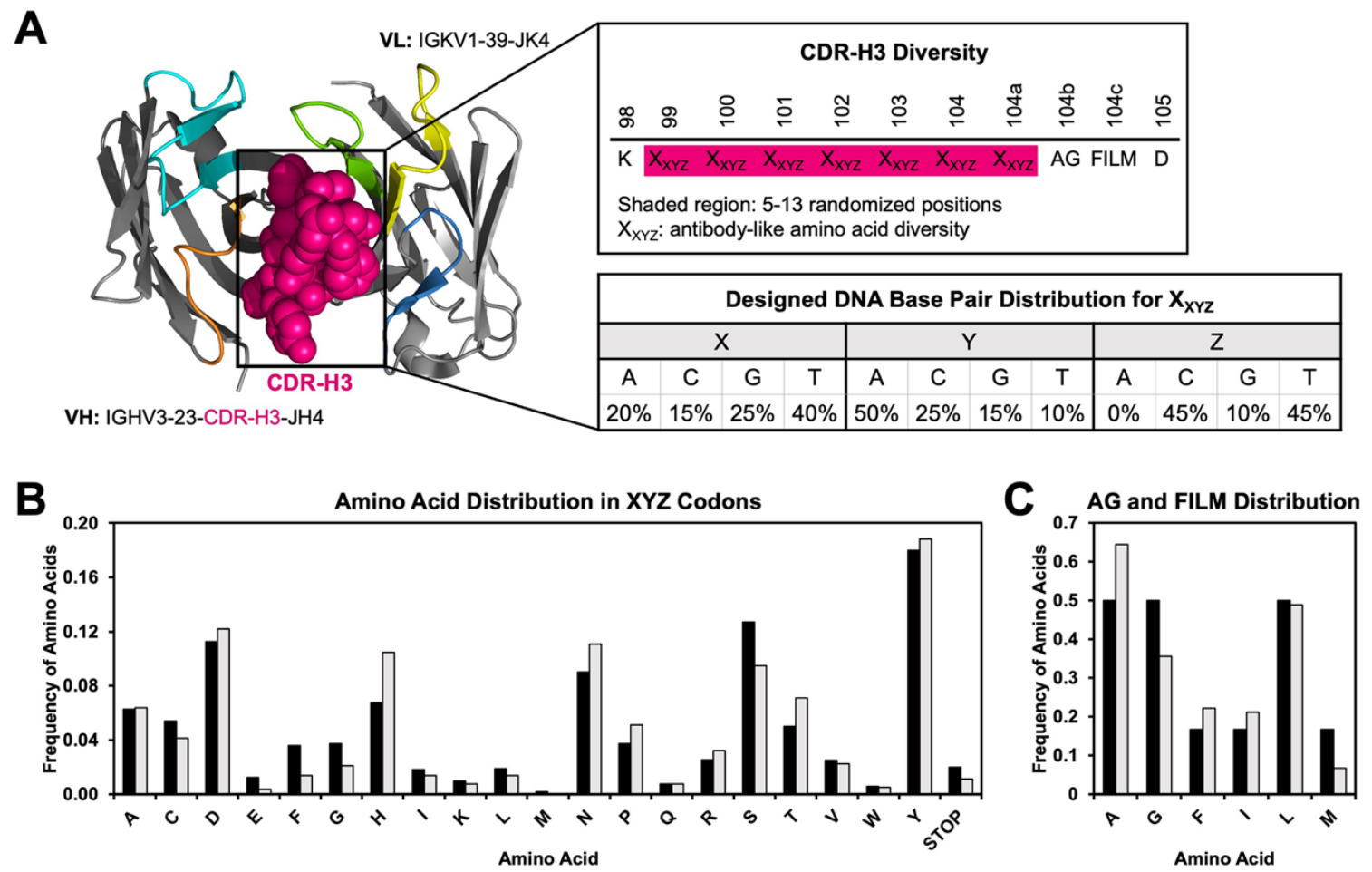

Predicted (based on library design) $\quad \square$ Observed (based on sequencing)

Figure 1. CDR-H3 library design and characterization. A) Antibody framework and CDR-H3 diversification scheme. Antibody structure generated using PyMol with PDB ID 1FVC. ${ }^{11}$ B) Predicted and observed frequencies of amino acid distribution in the diversified region of CDR-H3. C) Predicted and observed frequencies of amino acid distribution in the $A / G$ and $F / / / L / M$ positions in CDR-H3.

We prepared the CDR-H3 library as a series of sublibraries, each comprising a separate loop length of CDR-H3 within the range of 9 to 17 amino acids (Figure 1A) using homologous recombination yeast display library preparation. ${ }^{72-73}$ The theoretical diversity of the sublibraries 
range between $3.06 \times 10^{9}$ (loop length 9 ) and $8.62 \times 10^{22}$ (loop length 17 ) for a total possible diversity of $8.80 \times 10^{22}$ (Table 1 ). Due to library size restrictions in yeast display systems, we aimed for a total of approximately $1 \times 10^{9}$ transformants, distributed equally into each of the nine sublibraries. The actual number of transformants we obtained, which totaled to $1.36 \times 10^{9}$ for the entire library, was determined by plating samples of transformed cells from each sublibrary (Table 1). The observed percentage of truncated clones was determined experimentally by evaluating the fraction of clones lacking a C-terminal c-Myc tag using flow cytometry (SI Figure 1). As expected, the number of observed truncated clones increased with the number of " $X Y Z$ " codons since each additional " $X Y Z$ " codon introduces an additional possibility of a stop codon occurring in the diversified CDR-H3 loop that would truncate the scFv. The data also indicates that the observed percentage of truncated clones determined via flow cytometry was higher than the expected percentage of truncated clones in each sublibrary (SI Figure 1B). We attribute this observation to the fact that the expected truncation frequency only takes into account the probability of a stop codon occurring in the "XYZ" codon and does not predict insertions or deletions that could occur throughout the scFv leading to a truncation. In sequencing analysis of 95 clones (see also below), we observed that 4 clones contained a frameshift mutation in the scFv outside CDR-H3 (SI Table 4). Based on our flow cytometry analysis of a $22.4 \%$ truncation rate within the full library, we estimate that approximately $1.1 \times 10^{9}$ full-length transformants are present in the full CDR-H3 library (Table 1).

Table 1. Characterization of CDR-H3 library. Theoretical Diversity and Predicted Truncated Clones were calculated for each sublibrary based on library design. Number of Transformants was determined by colony counting, and Observed Truncated Clones was determined by flow cytometry (see Materials and Methods for details).

\begin{tabular}{|l|c|c|c|c|}
\hline Sublibrary & $\begin{array}{c}\text { Theoretical } \\
\text { Diversity }\end{array}$ & $\begin{array}{c}\text { Number of } \\
\text { Transformants }\end{array}$ & $\begin{array}{c}\text { Predicted Truncated } \\
\text { Clones (\%) }\end{array}$ & $\begin{array}{c}\text { Observed Truncated } \\
\text { Clones (\%) }\end{array}$ \\
\hline CDR9 & $3.06 \times 10^{9}$ & $1.20 \times 10^{8}$ & 9.60 & 18.2 \\
\hline CDR10 & $1.47 \times 10^{11}$ & $1.37 \times 10^{8}$ & 11.4 & 24.2 \\
\hline CDR11 & $7.04 \times 10^{12}$ & $1.52 \times 10^{8}$ & 13.2 & 20.4 \\
\hline CDR12 & $3.38 \times 10^{14}$ & $1.50 \times 10^{8}$ & 14.9 & 25.0 \\
\hline
\end{tabular}




\begin{tabular}{|l|c|c|c|c|} 
CDR13 & $1.62 \times 10^{16}$ & $1.80 \times 10^{8}$ & 16.6 & 24.6 \\
\hline CDR14 & $7.79 \times 10^{17}$ & $1.52 \times 10^{8}$ & 18.3 & 26.8 \\
\hline CDR15 & $3.74 \times 10^{19}$ & $1.22 \times 10^{8}$ & 19.9 & 30.3 \\
\hline CDR16 & $1.80 \times 10^{21}$ & $2.30 \times 10^{8}$ & 21.5 & 29.4 \\
\hline CDR17 & $8.62 \times 10^{22}$ & $1.20 \times 10^{8}$ & 23.1 & 30.8 \\
\hline Full Library & $8.80 \times 10^{22}$ & $1.36 \times 10^{9}$ & - & 22.4 \\
\hline
\end{tabular}

We validated the CDR-H3 library design by sequencing a sample of 8-12 clones from each sublibrary, for a total of 95 clones. Each of these clones had a unique CDR-H3 sequence, indicating that the library contains many unique transformants (SI Table 4). The distribution of

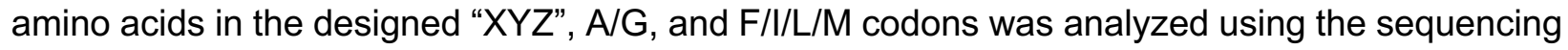
data from clones containing no frameshift mutations. The "XYZ" codon was designed based on Woldring et $\mathrm{al}^{69}$ with the predicted amino acid frequencies shown in Figure $1 \mathrm{~A}$ and nucleotide frequencies shown in SI Figure 2. Observed frequencies of each amino acid and nucleotide were found to be similar to the predicted frequencies. The most notable differences between designed and observed amino acid frequencies at positions encoded by "XYZ" codons were with respect to the observed frequencies of histidine, asparagine, threonine, and serine (Figure 1B). We also investigated the distribution of amino acids at positions encoded by the $A / G$ and $F / I / L / M$ codons and observed some differences between predicted and observed frequencies (Figure 1C). While amino acids found at $A / G$ and F/I/L/M positions deviated further from their predicted frequencies compared to the amino acid deviations found at "XYZ" codons, this could be due to the smaller number of $A / G$ and $F / / / L / M$ codons included sequenced (one per library clone compared to 5-13 per library clone for "XYZ" codons). Overall, our sequencing and flow cytometry data confirmed that the library contained the diversity that was expected based on design.

\section{Library Screening and Isolation of Binders}

Once we confirmed that the CDR-H3 library consisted of clones that were expected based on the "XYZ" codon design, we performed a series of proof-of-principle enrichments ${ }^{74-76}$ to 
determine whether this library contained binding clones capable of binding to structurally related IgGs from donkey, bovine, and rabbit (Figure 2 and SI Figure 3). The depicted results come from enrichments consisting of magnetic bead enrichments for four rounds and then a single round of fluorescence-activated cell sorting (FACS). Each round of magnetic bead sorting started with depletions against streptavidin-coated Dynabeads, beads precomplexed with biotin, and beads precomplexed with the murine IgG TA99 (see Materials and Methods for details). Then, the remaining cells were subjected to enrichments with target antigen-coated beads, grown to saturation, and analyzed for binding to the IgG by flow cytometry; progress in the enrichments was confirmed by this analysis after each round (Figure 2 and SI Figure 3). After four rounds of bead sorting and one round of FACS, the majority of cells in the enriched populations exhibited binding to the target IgG in flow cytometry experiments (Figure 2 and SI Figure 3). We then isolated and sequenced individual clones from the enriched populations targeting donkey lgG, bovine $\lg$, and rabbit $\lg$. From this sequencing, we identified several unique clones that appeared to be able to bind to their respective target antigens as evaluated by flow cytometry. Since antigen sorts were performed with biotinylated lgGs, competition studies were performed to identify clones binding to epitopes present on the native antigen while eliminating clones that bind preferentially to the biotinylated form of the antigen (SI Figures 4 and 5). Our competition data indicate that we identified both clones that bind to the authentic antigens and clones only capable of binding to biotinylated forms of the antigens. While the identification of clones that bind to biotinylated forms of antigens indicates that our library contains clones binding to multiple epitopes, the majority of these clones were not considered further after the competition experiments. The CDR-H3 sequences of clones capable of binding to the unmodified forms of the IgGs are listed in Table 2. Together, the screening results demonstrate the isolation of unique protein binders to donkey, bovine and rabbit IgG using the CDR-H3 library, despite limiting amino acid diversity to just the CDR-H3 region. 
Donkey lgG Sort 1

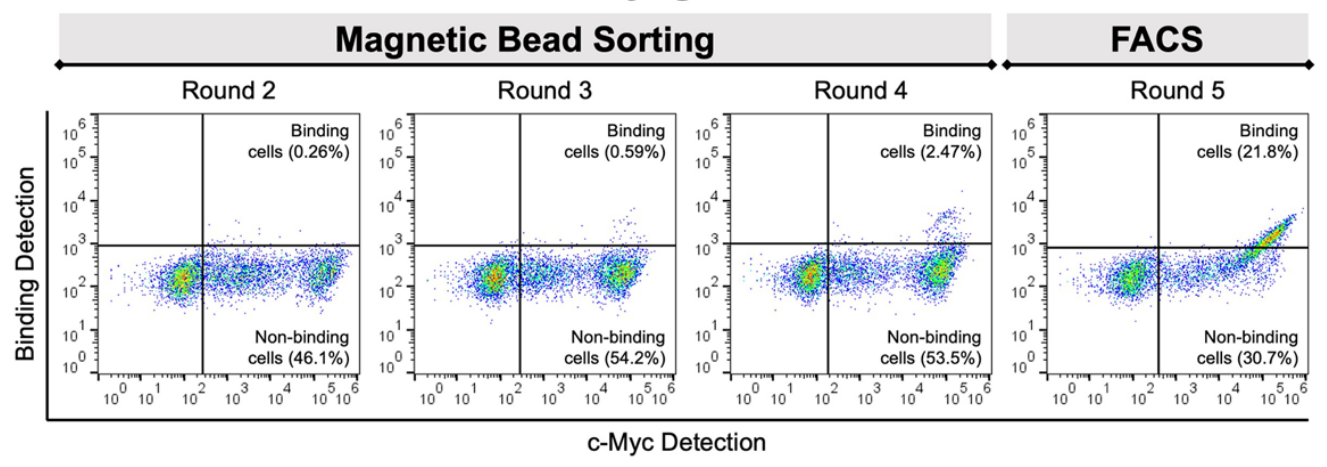

Figure 2. CDR-H3 library sorting against biotinylated donkey lgG. Binders were isolated using four rounds of magnetic bead sorting followed by one round of fluorescence-activated cell sorting (FACS). During FACS, cells were treated with $50 \mathrm{nM}$ biotinylated IgG and the population sorted for cells exhibit both fulllength display (c-Myc positive) and donkey lgG binding.

\section{Characterization of Isolated Binders}

In order to fully understand the properties of the scFvs isolated from this minimalist library, clones capable of binding to their target animal IgGs (donkey, bovine or rabbit) were further investigated for binding specificity as well as binding affinity (Figure 3 and Table 2). To determine the specificity of the isolated antibody fragments, we used a series of clones capable of binding to nonbiotinylated antigens as described above (SI Figure 4 and 5): two donkey IgG-binding scFvs (Donkey1.1 and 1.2), three bovine IgG-binding scFvs (Bovine2.1, 2.2, and 2.3) and one rabbit IgG-binding ScFv (Rabbit1.1). Since biotinylated forms of the lgGs were used for this assay, we also included one scFv that exhibited some amount of binding against only biotinylated form of donkey IgG (Donkey1.3) as a control for crossreactivity. ScFvs were displayed on the yeast surface and treated with IgGs to evaluate binding specificity. Figure $3 \mathrm{~A}$ and SI Figure 6 depict the results of flow cytometry experiments in which the set of scFvs was tested for binding against each of the three animal IgGs. At a fixed concentration of $200 \mathrm{nM} \operatorname{lgG}$, assays with both Donkey1.2 and Donkey1.3 result in binding signal when any of the three $\lg G$ s are used as the antigen. This is as expected for Donkey1.3 based on its preference for biotinylated antigen (SI Figure 4 and 5). Donkey1.2, which binds only nonbiotinylated donkey IgG, appears to exhibit 
crossreactivity against multiple IgGs. While the highest levels of IgG detection occur against donkey lgG, there is also measurable detection of bovine and rabbit lgG binding. In contrast, Donkey1.1, all three bovine IgG binders, and Rabbit1.1, appear to exhibit specificity to their target antigens based on the flow cytometry evaluations (Figure 3A and SI Figure 6); for all of these clones, binding signal is detected only against the cognate antigen. These experiments demonstrate that a single CDR loop is sufficient to yield binding proteins that discriminate between closely related antigens.
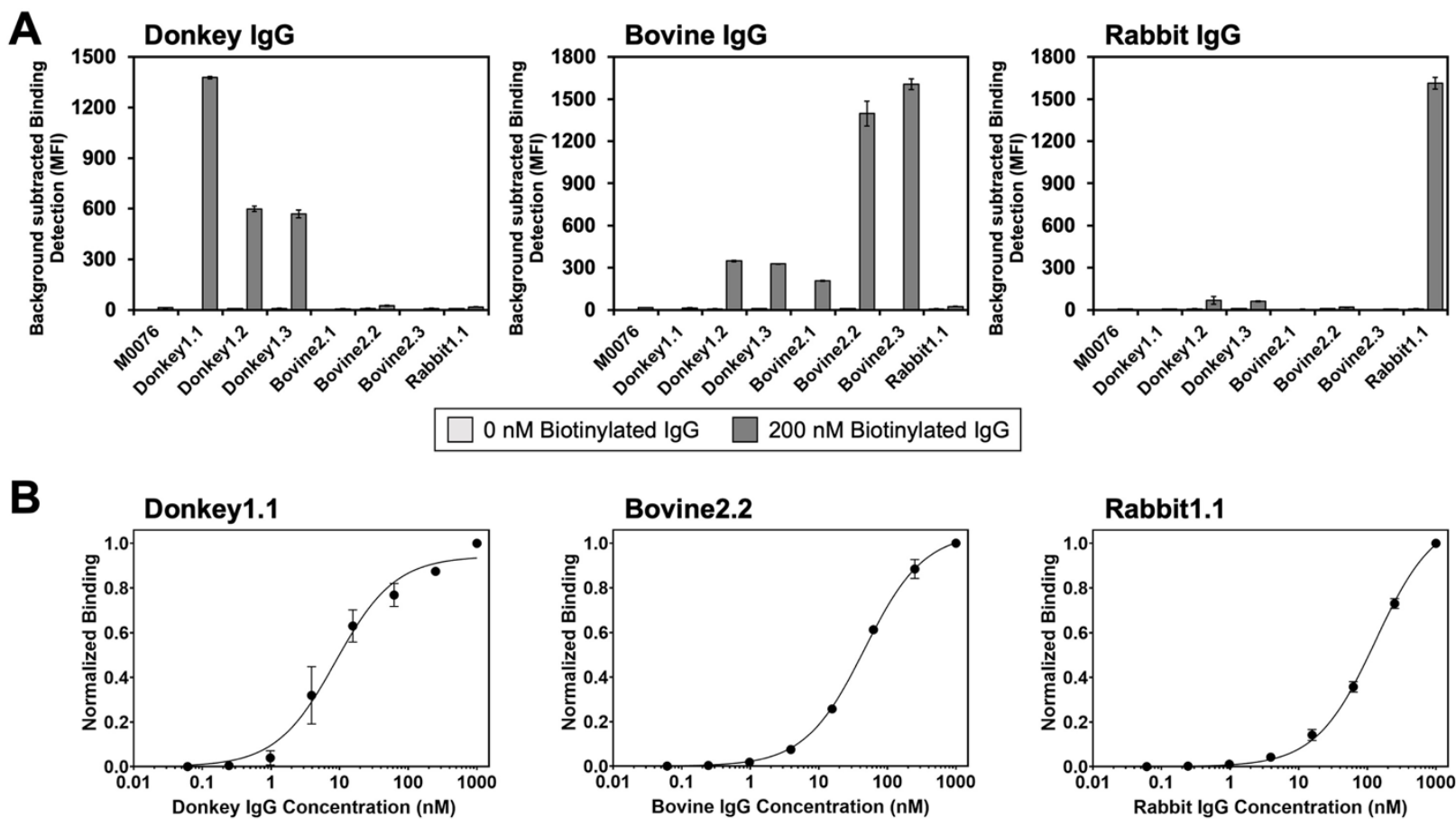

Figure 3. Binding characterizations of wild-type scFvs isolated from the library. A) Analysis of antigen specificity via crossreactivity assays. B) Binding titrations for the best performing wild-type clones. ScFvs were treated with different concentrations of biotinylated IgG (starting from $1 \mu \mathrm{M}$ with subsequent 4-fold dilutions) and labeled to detect biotinylated antigens. All characterizations were performed in technical triplicate, with error bars representing standard error (see Materials and Methods for details regarding normalization and analysis).

We also estimated the apparent affinities of the five antibody fragments exhibiting antigen specificity on the yeast surface (Table 2 and SI Figure 7; due to the off-target binding observed for Donkey1.2 and Donkey1.3, these scFvs were not considered further). Three of these scFvs (Donkey1.1, Bovine2.2, and Bovine2.3) exhibit relatively strong affinities, with Donkey1.1 
possessing an apparent $K_{D}$ of $8.75 \mathrm{nM}$ (95\% confidence interval: $\left.6.65-11.50 \mathrm{nM}\right)$ and two of the bovine IgG clones possessing $\mathrm{K}_{\mathrm{D}} \mathrm{s}$ in the doubt digit nanomolar range (Table 2). The other clones characterized here exhibited more modest affinities, although even for a comparatively weak binder such as Bovine $2.1\left(\mathrm{~K}_{\mathrm{D}}=746 \mathrm{nM}, 95 \%\right.$ confidence interval $\left.620-915 \mathrm{nM}\right)$, binding is readily detectable in flow cytometry-based assays. The affinities of clones isolated from this single-CDR antibody library are consistent with or stronger than the affinities of clones isolated from other libraries in which amino acid diversity is restricted to a single loop, ${ }^{27,66}$ confirming that a single loop is sufficient to support nanomolar binding affinities. With these simple, but specific, binding clones in hand, we investigated strategies for adding functionality to these antibodies via ncAA substitution and subsequent modifications based on the distinct side chain chemistries.

Table 2. Properties of clones isolated from CDR-H3 library. Each scFv was isolated by sorting against the target antigen, and binding affinity against biotinylated forms of these IgGs was determined from binding titrations using a saturation binding model on GraphPad Prism (see Materials and Methods for details).

\begin{tabular}{|c|c|c|c|c|}
\hline Clone & CDR-H3 Sequence & $\begin{array}{l}\text { CDR-H3 Loop } \\
\text { Length }\end{array}$ & $\begin{array}{c}\text { Target } \\
\text { Antigen }\end{array}$ & $\mathrm{K}_{\mathrm{D}}(95 \% \mathrm{Cl}), \mathrm{nM}$ \\
\hline Donkey1.1 & KYDKTHHNPDYALD & 14 & Donkey lgG & $8.75(6.65-11.50)$ \\
\hline Bovine2.1 & KHYDPYDDYNFYFGFD & 16 & \multirow{3}{*}{ Bovine IgG } & $746(620-915)$ \\
\hline Bovine2.2 & KTYYDNSYAYTALD & 14 & & $46.1(43.1-49.3)$ \\
\hline Bovine2.3 & KSSIYDGYMYGLD & 13 & & $38.4(33.4-44.2)$ \\
\hline Rabbit1.1 & KYNYHHPFYSYDAFD & 15 & Rabbit IgG & $131(121-142)$ \\
\hline
\end{tabular}

\section{Effects of Noncanonical Amino Acid Incorporation}

Binding affinity and protein display. In order to utilize the enhanced chemical diversity accessible through noncanonical amino acids, we sought to incorporate ncAAs without disrupting antibody function in the process. As such, we first investigated the effects of noncanonical amino acid incorporation on antigen binding and full-length scFv display to determine the feasibility of this strategy. We made ncAA substitutions at multiple positions within scFv variable domains using stop codon suppression to genetically encode ncAAs in response to the amber (TAG) stop codon. $^{62-64,}$ 77-83 Plasmids encoding the donkey IgG binder Donkey1.1, bovine lgG binders 
Bovine2.1 and Bovine2.2, and the rabbit IgG binder Rabbit1.1 were mutated to contain a TAG codon at one of three sites: position 93 in the light chain of the ScFv (located in CDR-L1; originally serine (TCT)), position 31 in the heavy chain of the scFv (located within CDR-H1; originally serine $(A G C)$ ) or position 54 in the heavy chain of the ScFv (located within CDR-H2; originally serine (TCT)). These positions will be referred to as $\mathrm{L} 93, \mathrm{H} 31$ and $\mathrm{H} 54$, respectively, and resulting mutants will be referred to as the L93TAG, H31TAG and H54TAG variants of the parent scFvs. These positions were chosen based on their locations outside of CDR-H3 but within other CDRs that can be involved in antigen binding, ${ }^{84}$ and based on the predicted surface accessibility of native residues using GetArea calculations in several antibody fragment structures (PDB 3AUV, ${ }^{85}$ $2 \mathrm{KH} 2,{ }^{86} 3 \mathrm{~K} 2 \mathrm{U},{ }^{87}$ and $\left.5 \mathrm{JYL}^{88}\right)$. NcAAs were incorporated using constitutively expressed orthogonal translation systems (OTSs; Figure 4A), which in this work are previously described aminoacyl-tRNA synthetase/tRNA (aaRS/tRNA) pairs. ${ }^{62-64}$ Figure 4B depicts the ncAAs used in this work: $\quad p$-azido-L-phenylalanine $(\mathrm{AzF}), \quad p$-azidomethyl-L-phenylalanine $\quad(\mathrm{AzMF}), \quad p$ propargyloxyphenylalanine (OPG) and H-L-Lys(EO-N3)-OH (LysN3). These ncAAs were incorporated using aaRSs derived from either the E. coli tyrosyl-tRNA synthetase or the E. coli leucyl-tRNA synthetase known as AcFRS (AzF and OPG), LeuOmeRS (OPG and AzMF), or LeuRS BH5 T252A (LysN3). These aaRSs were paired with corresponding suppressor tRNA $_{C U A}{ }^{\text {Tyr }}$ or tRNACUA ${ }^{\text {Leu }} 62-63,89-90$ (note: aaRS/tRNA pairs will be referred to by specifying only the aaRS for the remainder of this manuscript). Yeast cotransformed with TAG-containing scFv constructs and stop codon suppression machinery were induced in the presence of ncAAs to display substituted clones on the yeast surface, treated with a $200 \mathrm{nM}$ concentration of the respective target $\lg$, and analyzed for display levels and antigen binding detection via flow cytometry. 


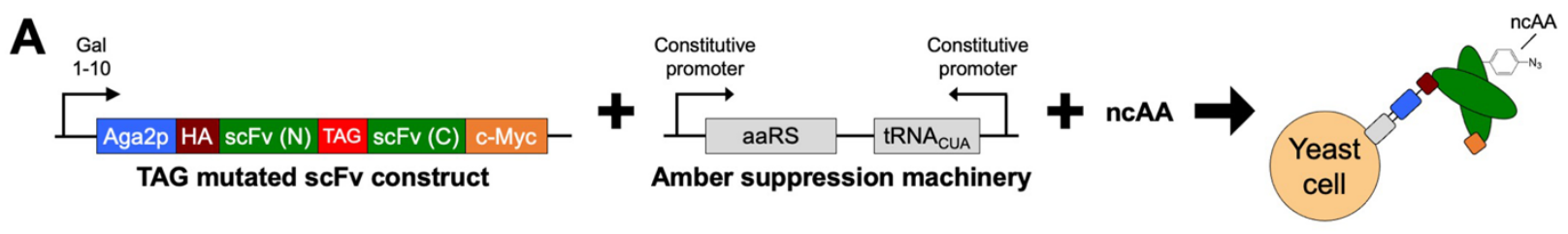

B

C Biotinylated IgG Binding Detection

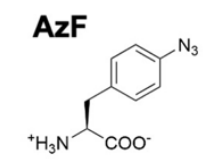<smiles>CN(C)c1ccc(N)cc1N</smiles>
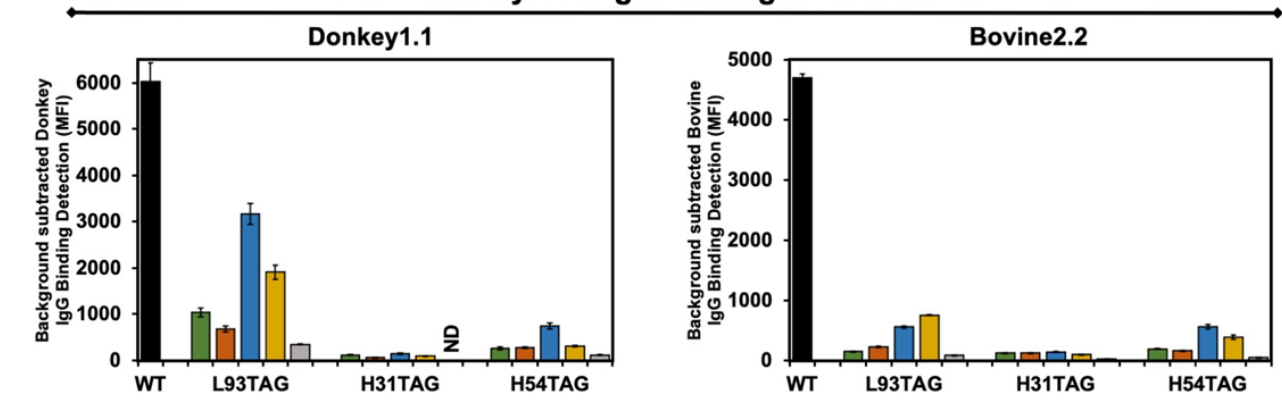

D<smiles>C=COc1ccc(CNC(=O)OCc2ccccc2)cc1</smiles><smiles>CNC(=O)CCCCNC(=O)OCCN</smiles>
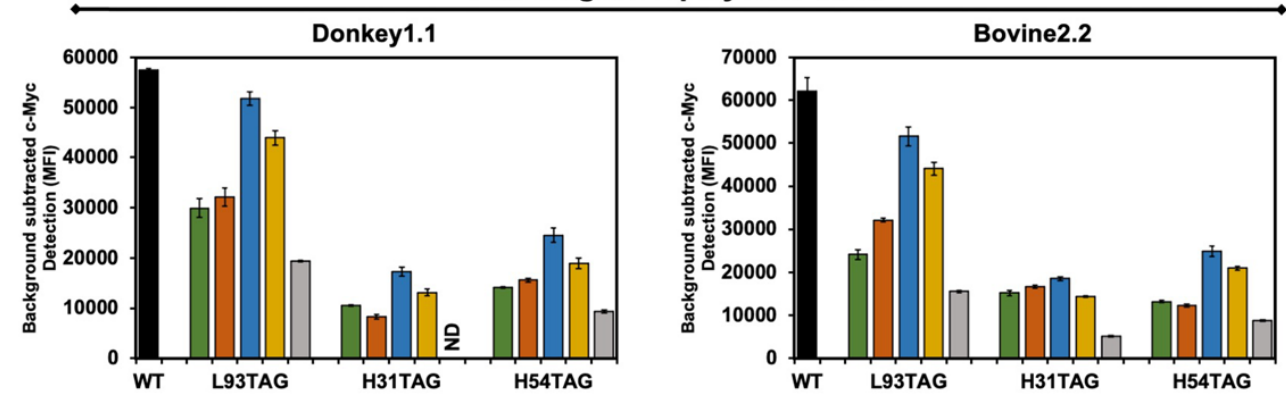

$\varnothing / \varnothing \square$ AcFRS/AzF $\square$ AcFRS/OPG $\square$ LeuOmeRS/OPG $\square$ LeuOmeRS/AzMF $\square$ LeuRS BH5 T252A/LysN3

E

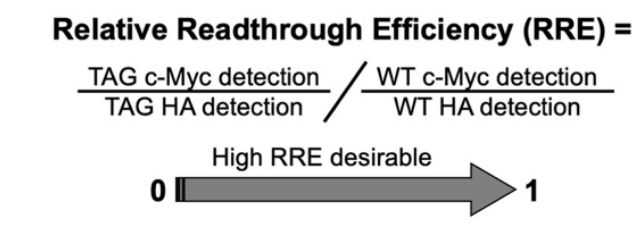

Maximum Misincorporation Frequency (MMF) = RRE without ncAA / RRE with ncAA
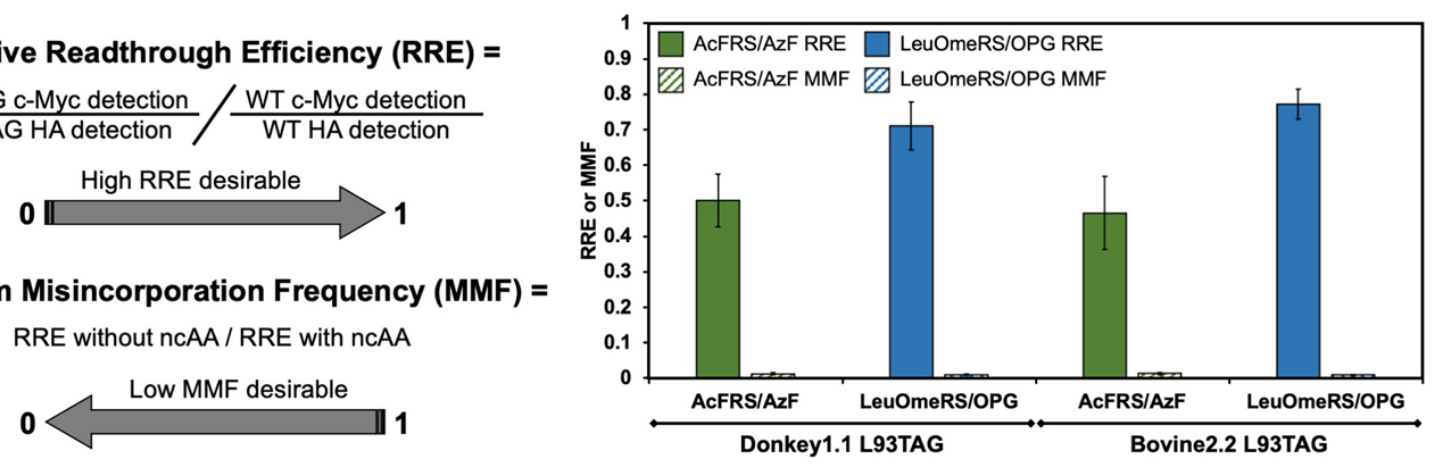

Figure 4. Evaluation of noncanonical amino acid (ncAA) substitution effects on antigen binding and fulllength display. A) NcAA incorporation strategy using amber suppression machinery. B) NcAAs used in this study: $\quad p$-azido-L-phenylalanine $\quad(\mathrm{AzF}), \quad p$-azidomethyl-L-phenylalanine $\quad(\mathrm{AzMF}), \quad p$ propargyloxyphenylalanine (OPG) and H-L-Lys(EO-N3)-OH (LysN3). C) Binding analysis of Donkey1.1 (left) and Bovine2.2 (right) clones substituted with ncAAs using $200 \mathrm{nM}$ biotinylated donkey or bovine IgG, respectively. D) Full-length display detection of Donkey1.1 (left) and Bovine2.2 (right) clones via c-Myc epitope tag labeling. For data shown in both panels $C$ and $D$, values here are background-subtracted median fluorescence intensity (MFI) values from experiments performed in technical triplicate (see Materials and Methods for details). E) Relative readthrough efficiency (RRE) and maximum misincorporation frequencies (MMF) of ncAA substitutions in Donkey1.1 L93TAG and Bovine 2.2 L93TAG. AzF and OPG were inserted using the AcFRS and LeuOmeRS aaRSs, respectively. See Materials and Methods for calculation details. ND, not determined. 
Noncanonical amino acid substitutions resulted in varying effects on antigen binding for the four clones analyzed here (Figure 4C and SI Figures 8-10). Three out of the four clones (Donkey1.1, Bovine 2.1, and Bovine 2.2) retained binding function after almost every ncAA substitution at position L93. This tolerance for substitutions at L93 is in line with the work of Jespers et al in which cysteine introduced at L93 of a phage displayed antibody library was found to be well-tolerated ${ }^{60}$. Three out of four clones (Donkey1.1, Bovine2.2, and Rabbit1.1) retained binding function after ncAA substitution at $\mathrm{H} 54$, while substitutions at position $\mathrm{H} 31$ tended to result in lower antigen binding levels. Two clones exhibited broad tolerance of ncAA substitutions: in Donkey1.1 and Bovine2.2, almost every combination of aaRS/tRNA, ncAA, and substitution site tested resulted in detection of donkey $\lg G$ and bovine lgG binding, respectively (Figure 4C and SI Figure 8). With the exception of LysN3 substitution at position $\mathrm{H} 31$, all flow cytometry dot plots clearly indicate a population of cells binding the target antigen (SI Figure 8). Several substitutions in Donkey1.1 and Bovine2.2 resulted in moderate to high levels of antigen detection at $200 \mathrm{nM}$ concentration, with the highest antigen detection being OPG incorporation with LeuOmeRS in Donkey1.1 (antigen detection levels of approximately $50 \%$ of wild-type). On the other hand, ncAA substitutions at position $\mathrm{H} 31$ led to antigen binding levels of less than $10 \%$ of wild-type levels for all ncAAs tested in Donkey1.1 and Bovine2.2. Two clones exhibited poor tolerance for ncAA substitutions: ncAA substitutions in clones Bovine2.1 and Rabbit1.1 resulted in greatly diminished or undetectable antigen binding for all substitutions investigated (SI Figures 8-10). These results are particularly surprising for Rabbit1.1, as the parent clone exhibits moderate affinity towards rabbit $\lg G\left(K_{D}=131 \mathrm{nM}, 95 \%\right.$ confidence interval 121- $\left.142 \mathrm{nM}\right)$. On the other hand, the loss of antigen detection in the case of ncAA-substituted Bovine2.1 clones is consistent with the weak affinity of the parent clone $\left(K_{D}=746 \mathrm{nM}, 95 \%\right.$ confidence interval $\left.620-915 \mathrm{nM}\right)$.

Differences in antigen binding following ncAA substitution indicate that these four clones exhibit a range of tolerances for ncAA substitutions in positions located outside of CDR-H3. To 
address one potential reason for these variable tolerances, we examined how ncAA incorporation efficiency affects antigen binding. All ncAA substitutions investigated decreased c-Myc detection levels; detected display levels range from approximately $10 \%$ to $90 \%$ of parent clone display levels (Figure 4D, SI Figure 9). Decreases in display are expected based on known ncAA incorporation efficiencies of the orthogonal translation systems (OTSs) used here. ${ }^{63}$ Since stop codon context can have large effects on ncAA incorporation efficiency and fidelity ${ }^{62-64}$, we determined relative readthrough efficiency (RRE) and maximum misincorporation frequency (MMF) for selected clones at the L93 site (Figure 4E; RRE and MMF are measures of ncAA incorporation efficiency and fidelity, respectively). For the variants Donkey1.1 L93TAG and Bovine2.2 L93TAG prepared with the aaRS/tRNA + ncAA combinations of AcFRS + AzF and LeuOmeRS + OPG, we determined RRE to be in the range of 0.47-0.77 (Figure 4E and SI Table 10). These values indicate that ncAA insertions in these clones at position L93 are moderately efficient, possessing increased RRE values compared to the values obtained with the same OTS + ncAA combinations with our previously described yeast display reporter system. ${ }^{63}$ Measurements of MMF resulted in almost undetectable levels of misincorporation of canonical amino acids, confirming the apparent fidelity of these incorporation events. Thus, while inserting ncAAs in these displayed clones is a relatively efficient process, some loss of display levels is expected based on diminished ncAA incorporation efficiency alone.

We surveyed changes in display levels in Donkey1.1 and Bovine2.2 and compared these changes to changes in antigen binding levels. We focused on two key sets of comparisons for a number of aaRS/tRNA + ncAA + insertion site combinations. The data shown in SI Figure 10A and 10B directly compares antigen binding and display levels observed for OPG-containing clones prepared with each of two aaRS/tRNA pairs (AcFRS and LeuOmeRS). As expected, clones prepared with LeuOmeRS exhibit higher antigen binding and display levels than clones prepared with AcFRS, confirming the role of display level in dictating antigen binding. However, 
we note that the decreases in binding levels observed are greater than corresponding decreases in display levels upon ncAA substitution. This trend also generally holds when comparing display levels and antigen binding levels in AzMF- and OPG-substituted clones prepared using the same LeuOmeRS (SI Figure 10C and 10D). These variants exhibit display levels within 10-20 percent of one another, but differences in antigen binding between the two variants approach as high as $50 \%$ in some cases. Taken together, these findings show that changes in display levels upon ncAA substitution directly account for only a portion of the changes in antigen binding levels measured in these studies.

Based on the observation that antigen binding levels in ncAA-substituted clones tend to decrease more substantially than corresponding display levels, we investigated the possibility that ncAA substitutions decrease apparent binding affinities. To address this question, we used yeastbased titrations to evaluate potential changes in antigen binding affinity following ncAA substitutions in Donkey1.1 and Bovine2.2 (SI Figure 12). Table 3 reports the $K_{D}$ values of ncAAsubstituted scFvs prepared with two aaRS/tRNA + ncAA combinations (AcFRS + AzF and LeuOmeRS + OPG) on the yeast surface and the fold change of the $K_{D}$ s from the corresponding observed wild-type $K_{D} S$. In general, OPG-containing clones exhibited relatively strong apparent affinities, with substitutions at L93 and H54 increasing apparent $K_{D}$ s by 3.9- to 6.4-fold compared to WT. In contrast, AzF substitutions at these positions were more poorly tolerated, resulting in increases in apparent $K_{D}$ s ranging from 7.2- to 24.4-fold. NcAA substitutions at position H31 of both Donkey1.1 and Bovine2.2 were not well-tolerated, resulting in increases in apparent $\mathrm{K}_{\mathrm{DS}}$ ranging from 11.9-fold to 64.1 -fold. Given that some of the resulting $K_{D} S$ for these clones are well above $200 \mathrm{nM}$, this helps to explain the low levels of antigen detection observed in comparison to changes in clone display levels (Figure 4C and D). We further confirmed this observation by noting that antigen binding levels of substituted clones increase when treated with $1 \mu \mathrm{M}$ antigen during titration experiments (SI Figure 13). These data suggest that ncAA substitutions, although 
located outside of the primary specificity-determining CDR-H3 loop, can weaken apparent clone affinities.

Table 3. Binding affinities of AzF- and OPG-containing variants of Donkey 1.1 and Bovine 2.2 clones. Affinities were determined from binding titrations with biotinylated IgGs using a saturation binding model on GraphPad Prism. Fold change from WT indicates the effect of ncAA incorporation on binding interactions between the scFv and IgGs (see Materials and Methods for details).

\begin{tabular}{|c|c|c|c|c|}
\hline Clone & aaRS/ncAA & $\begin{array}{c}\text { Target } \\
\text { Antigen }\end{array}$ & $\mathrm{K}_{\mathrm{D}}(95 \% \mathrm{Cl}), \mathrm{nM}$ & $\begin{array}{l}\text { Fold Change } \\
\text { from WT } \\
\left(\frac{\mathbf{K}_{\mathbf{D}(\mathrm{TAG})}}{\mathbf{K}_{\mathrm{D}(\mathrm{WT})}}\right)\end{array}$ \\
\hline \multirow{2}{*}{ Donkey1.1 L93TAG } & AcFRS/AzF & \multirow{6}{*}{ Donkey IgG } & $125(99-158)$ & 14.3 \\
\hline & LeuOmeRS/OPG & & $45.0(29.2-68.5)$ & 5.10 \\
\hline \multirow{2}{*}{ Donkey1.1 H31TAG } & AcFRS/AzF & & $561(517-610)$ & 64.1 \\
\hline & LeuOmeRS/OPG & & $365(335-399)$ & 41.7 \\
\hline \multirow{2}{*}{ Donkey1.1 H54TAG } & AcFRS/AzF & & $213(173-263)$ & 24.4 \\
\hline & LeuOmeRS/OPG & & $55.8(38.8-79.8)$ & 6.40 \\
\hline \multirow{2}{*}{ Bovine2.2 L93TAG } & AcFRS/AzF & \multirow{6}{*}{ Bovine IgG } & $332(273-406)$ & 7.20 \\
\hline & LeuOmeRS/OPG & & $179(155$ - 208) & 3.90 \\
\hline \multirow{2}{*}{ Bovine2.2 H31TAG } & AcFRS/AzF & & $546(474-633)$ & 11.9 \\
\hline & LeuOmeRS/OPG & & $601(515-708)$ & 13.0 \\
\hline \multirow{2}{*}{ Bovine2.2 H54TAG } & AcFRS/AzF & & $380(310-471)$ & 8.30 \\
\hline & LeuOmeRS/OPG & & $183(132-254)$ & 4.00 \\
\hline
\end{tabular}

Overall, these substitution studies demonstrate that some synthetic antibodies tolerate ncAA substitution at multiple substitution sites. Our data also indicate that the degree to which clones tolerate ncAA substitutions depends on the specific clone, substitution site, ncAA, and even the OTS-dependent display level of ncAA-containing clones. While further studies will be required to fully understand the molecular underpinnings of some of our observations, the ncAA substitution tolerances of Donkey1.1 and Bovine2.2 enabled us to examine how to exploit the unique chemical properties of the resulting clones in the following sections.

Click chemistry and its effects on binding. Genetically encoding azides or alkynes within antibodies enables the use of bioorthogonal chemistries to create well-defined conjugates, ${ }^{91-94}$ including conjugates on the yeast surface. ${ }^{53,62-63}$ Here, we investigated modifications to 
Donkey1.1 and Bovine2.2 clones containing ncAAs at positions L93 and H54 positions to determine clone reactivity and investigate the effects of chemical modifications on antigen binding (Figure 5). While most investigations of antibody conjugation strategies focus on sites located distal to antibody CDRs, the L93 and H54 sites used in this work are located directly within CDRL3 and CDR-H2, respectively; little is known about the effects of conjugations at these sites on antibody function. To evaluate both reactivity and subsequent binding, we used copper-catalyzed azide-alkyne cycloaddition (CUAAC) and strain-promoted azide-alkyne cycloaddition (SPAAC) reactions with biotin probes ${ }^{95-100}$ (Figure $5 \mathrm{~A}$ ). Biotin-alkyne and biotin-azide probes were used to evaluate CuAAC reactions with clones containing azide- and alkyne-containing ncAAs, respectively; biotin-dibenzocyclooctyne (DBCO) was used to evaluate SPAAC reactions. For all reactions reported here, we used the same set of empirically determined conditions for CuAAC and SPAAC reactions (SI Figures 15 and 16). For CuAAC, $100 \mu \mathrm{M}$ probe concentration led to maximum biotinylation within 15 minutes without detectable nonspecificity for a previously described model reporter protein containing $A z F ; 63$ we used these conditions for all CuAAC reactions (SI Figure 15). For SPAAC, reactions with $10 \mu \mathrm{M}$ biotin-DBCO at $4{ }^{\circ} \mathrm{C}$ suppressed nonspecific reactions, but we limited reaction times with biotin-DBCO to 2 hours based on the observation of biotinylation of WT Bovine2.2 beginning at 2 hours (SI Figure 16). 
Azide-containing ncAA

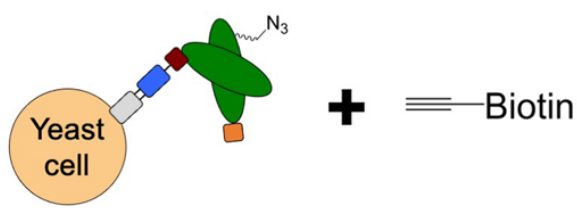

15 minutes, RT

1. CuSO4 + THPTA

2. Aminoguanidine

3. Sodium ascorbate

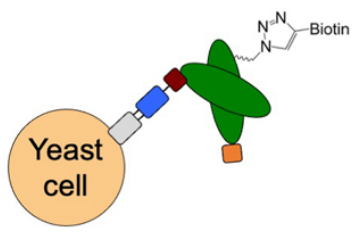

Alternatively, use alkyne-containing ncAA with Biotin-Azide

Strain Promoted Azide Alkyne Cycloaddition (SPAAC)
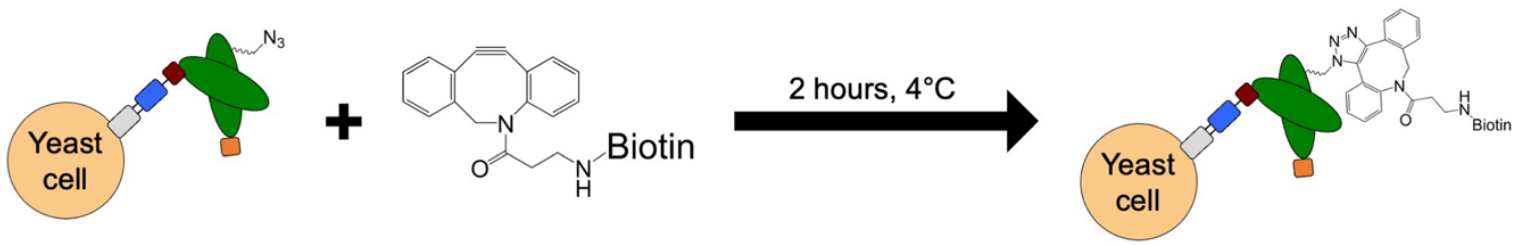

B

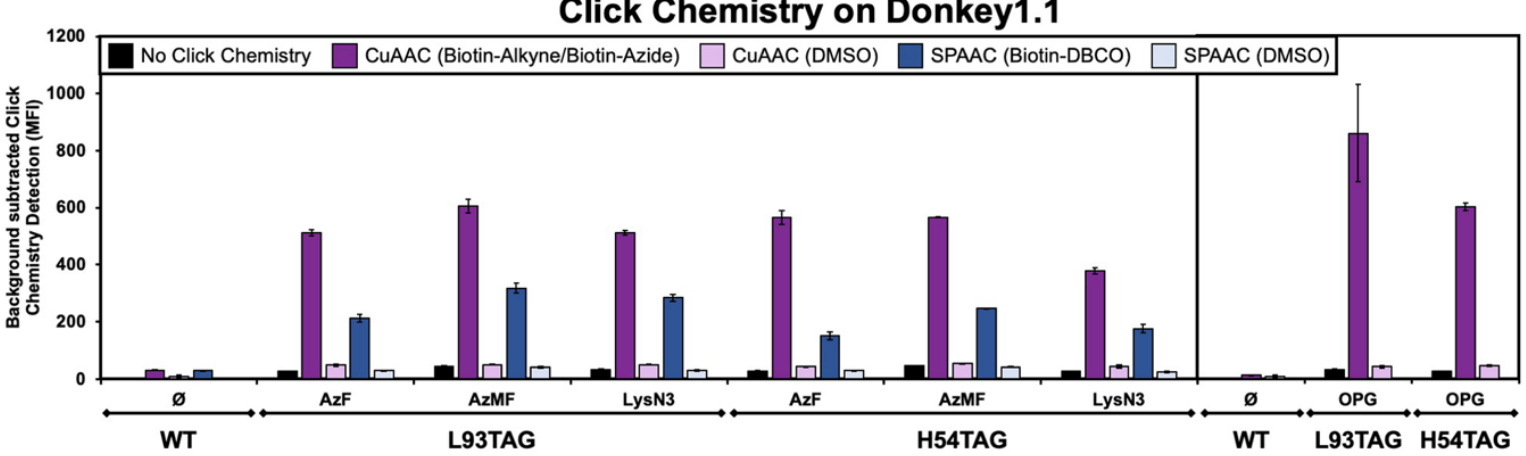

C

Post-Click Chemistry Donkey IgG Binding Detection

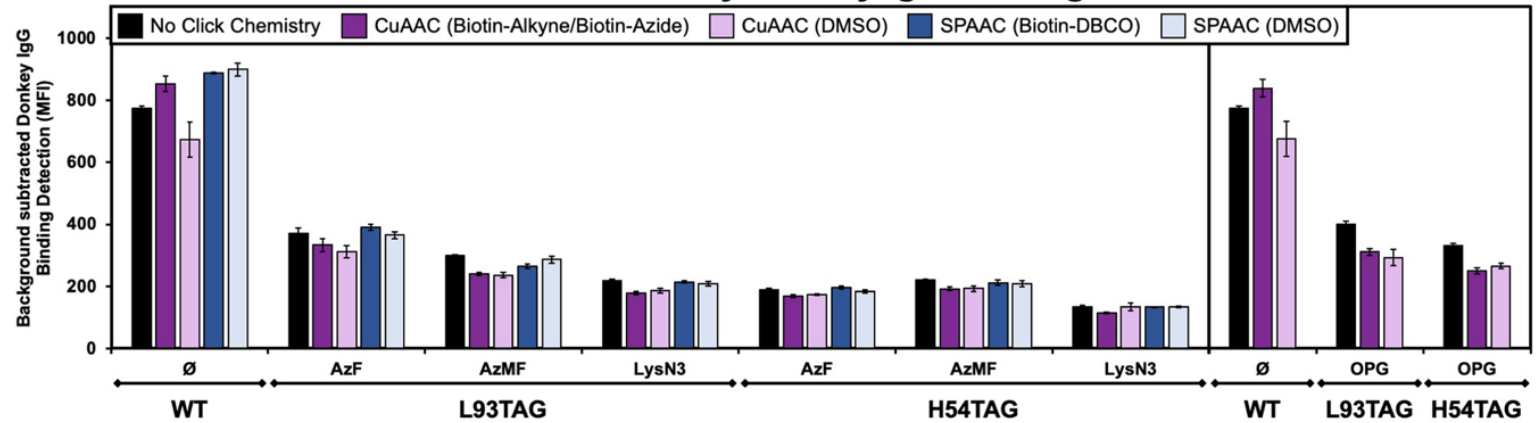

Figure 5. Click chemistry reactions and binding analysis of clones following reactions. A) Copper-catalyzed azide-alkyne cycloaddition (CuAAC) and strain-promoted azide-alkyne cycloaddition (SPAAC) reaction schemes to modify yeast-displayed scFvs. B) Evaluation of CuAAC and SPAAC reactions using reactive biotin probes. All samples were subjected to CuAAC or SPAAC conditions, with DMSO controls omitting reactive alkyne probes. C) Binding with $200 \mathrm{nM}$ donkey IgG after subjecting samples to CuAAC and SPAAC reactions. For data shown in both panels $B$ and $C$, values here are background-subtracted median fluorescence intensity (MFI) values from experiments performed in technical triplicate (see Materials and Methods for details). 
Every combination of aaRS, ncAA, substitution site, and clone resulted in substantial biotin detection for CUAAC and SPAAC, while all control reactions led to minimal or undetectable labeling (Figure 5 and SI Figures 17-21). For the combinations tested here, CuAAC reactions resulted in higher relative biotin detection levels than the corresponding SPAAC reactions (Figure $5 B$ ), which we attribute primarily to using SPAAC conditions that limit off-target reactions rather than maximizing yield. For Donkey1.1, scFvs containing azides (AzF, AzMF and LysN3) subjected to CuAAC all resulted in similar levels of biotin detection, whereas for Bovine2.2 clones substituted with AzMF exhibit higher levels of biotin detection than clones containing AzF or LysN3 (SI Figure 21). Some of these differences are likely the result of changes in the levels of full-length constructs that depend on the specific suppression machinery used.$^{63}$ As observed in Figure 4, LeuOmeRS (incorporating AzMF and OPG) appears to support high levels of full-length display, followed by moderate levels of display for AcFRS (incorporating AzF), and low levels of display with LeuRS BH5 T252A (incorporating LysN3). CuAAC reactions with OPG-substituted clones resulted in higher biotin detection levels than those observed with antibodies containing any of the azide groups considered here. Previous work has indicated that even subtle changes to reactants used in CuAAC can strongly influence reaction kinetics and product yield, ${ }^{101-102}$ consistent with our observations that changes in ncAA side chain result in changes in relative reaction levels under a fixed set of reaction conditions.

Following the click chemistry reactions, we evaluated whether the reactions affected scFv binding. We hypothesized that since our clones were engineered to contain only variable CDRH3 loops, installation of chemical groups in other CDRs should be well-tolerated. Following CuAAC or SPAAC reactions, yeast displaying scFvs were treated with $200 \mathrm{nM}$ target lgG (donkey IgG for Donkey1.1 and bovine lgG for Bovine2.2, respectively) and analyzed for antigen binding. For these experiments, antigen binding was detected by using anti-donkey or anti-bovine secondary antibodies to detect unmodified donkey or bovine IgG, respectively (since the click 
chemistry reactions result in the installation of a biotin moiety). Figure 5C and SI Figure 21 compare the binding levels between biotin-clicked, DMSO-clicked and non-clicked (control) samples. Based on the levels of IgG detected, neither installation of the biotin probes nor the CuAAC and SPAAC reaction conditions appear to affect binding levels for most of the clones analyzed (for the OPG-containing Bovine2.2 H54TAG clone however, we did observe decreased binding after CuAAC and SPAAC reactions (SI Figure 21), indicating that at least in this one instance, the addition of external chemical groups impacts binding). It is especially noteworthy that even samples treated with the bulky DBCO reagent retain similar levels of antigen binding to CuAAC samples for the clones considered in this study. These findings demonstrate that conjugations at the L93 and H54 positions are well-tolerated, consistent with the hypothesis that interactions with $\mathrm{CDR}-\mathrm{H} 3$ are driving binding interactions with the antigens. Overall, these studies demonstrate that scFvs containing ncAA substitutions positioned within CDRs can be chemically addressed using CuAAC and SPAAC on the yeast surface, and that the resulting conjugates retain binding function even upon installation of large moieties.

Covalent target engagement using UV mediated photo-crosslinking. Antibodies containing only canonical amino acids rarely engage in covalent interactions with target antigens. Given the well-known photo-crosslinking capabilities of $p$-azido-L-phenylalanine $(\mathrm{AzF}){ }^{103-111}$ we investigated whether presentation of AzF within synthetic antibodies could facilitate covalent crosslinking on the yeast surface and in solution (Figure 6). Figure 6A depicts our general strategy for evaluating covalent crosslinking on the yeast surface using a competition assay. In this threestep approach, scFvs are treated with unmodified IgGs to allow binding between the antigen and antibodies. Next, the samples are subjected to UV irradiation to initiate crosslinking. Lastly, the samples are incubated with a higher concentration of the $\operatorname{lgG}$, but this time using the biotinylated form. Flow cytometry-based biotin detection is then used to evaluate the extent to which the biotinylated form of the antigen has competed off the unmodified form (Figure 6A). Accompanying 
each of these steps are controls to elucidate wild-type and non-crosslinking behavior. These experiments provide a means of evaluating the extent to which AzF-substituted clones facilitate crosslinking on the yeast surface. 


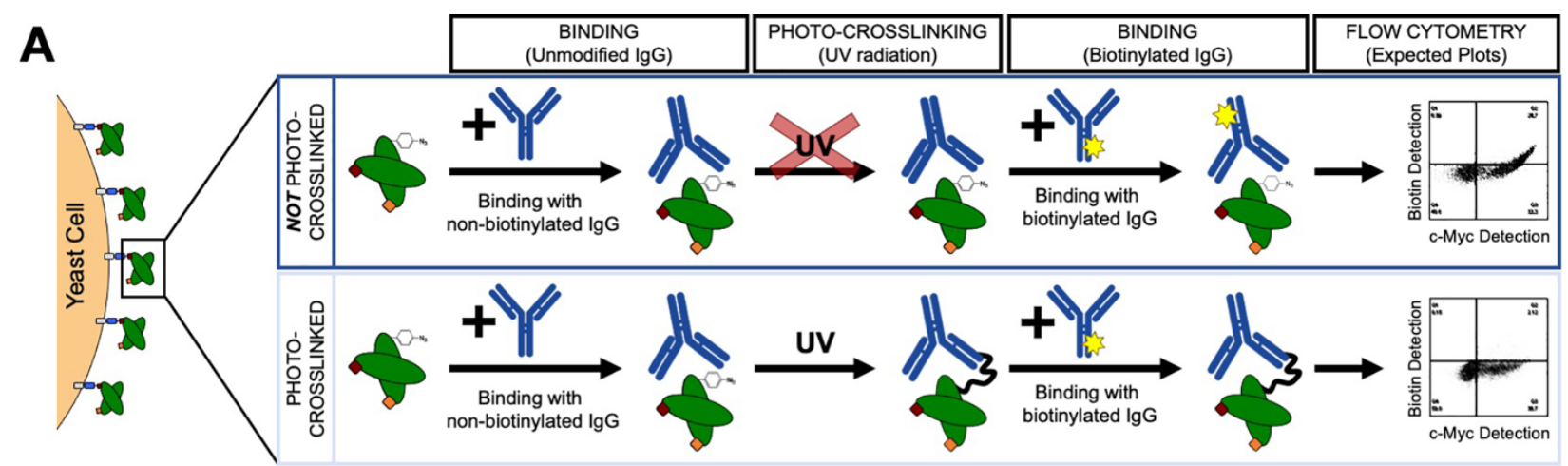

B

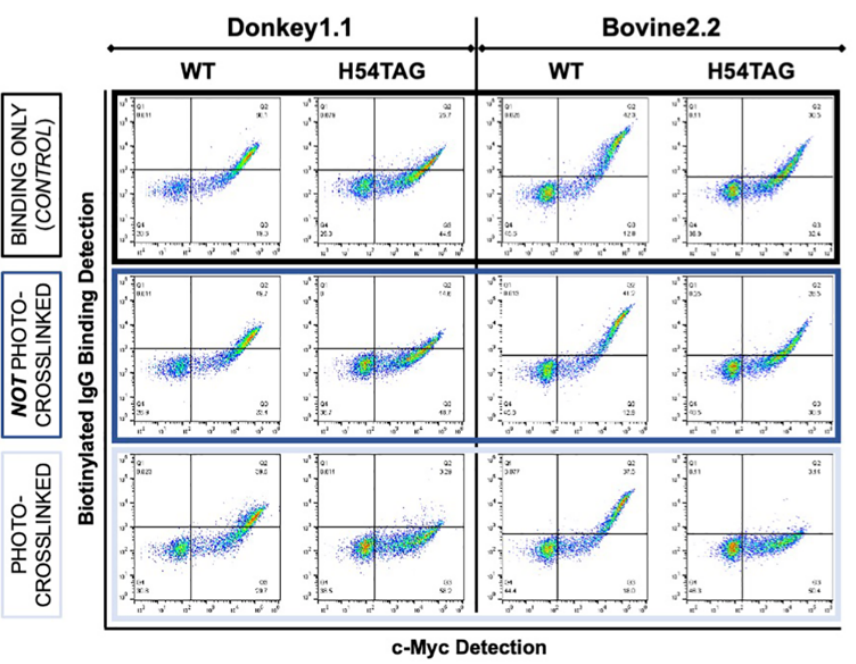

C

\section{Binding Fraction Exchanged $=$}

Apparent Percentage Binding (Sample)

Apparent Percentage Binding (Control)

Where:

Apparent Percentage Binding =

$$
\left(\frac{\text { Population Count }(\mathrm{Q} 2)}{\text { Population Count }(\mathrm{Q} 2+\mathrm{Q} 3)}\right) * 100
$$

D
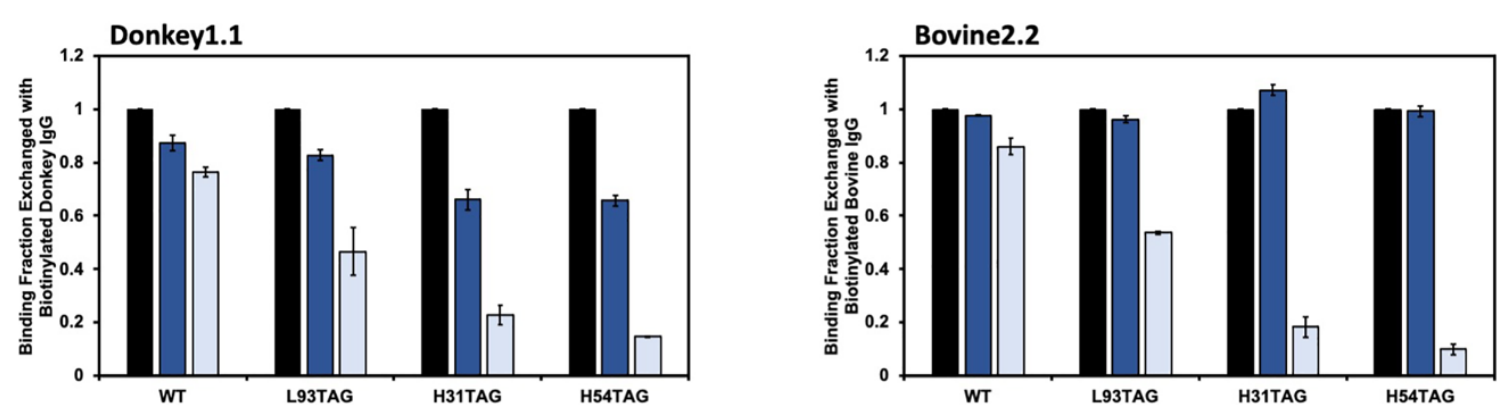

Binding Only (Control) $\square$ Not Photo-crosslinked $\square$ Photo-crosslinked

Binding Only (Control) $\square$ Not Photo-crosslinked $\square$ Photo-crosslinked

E

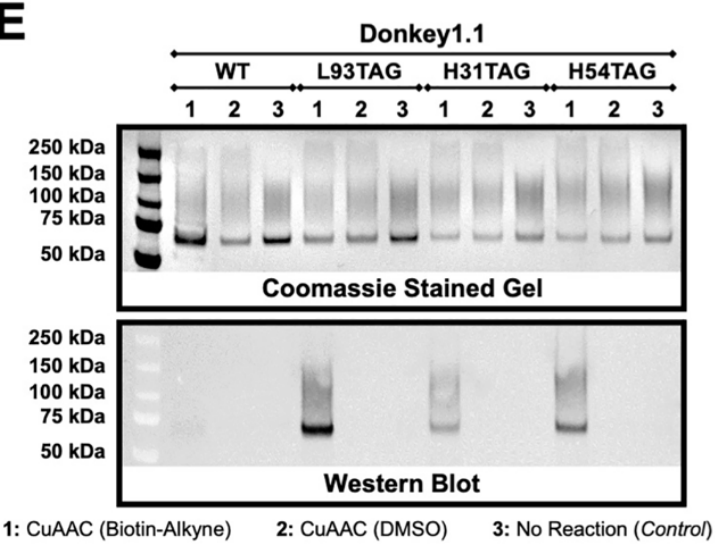

$\mathbf{F}$

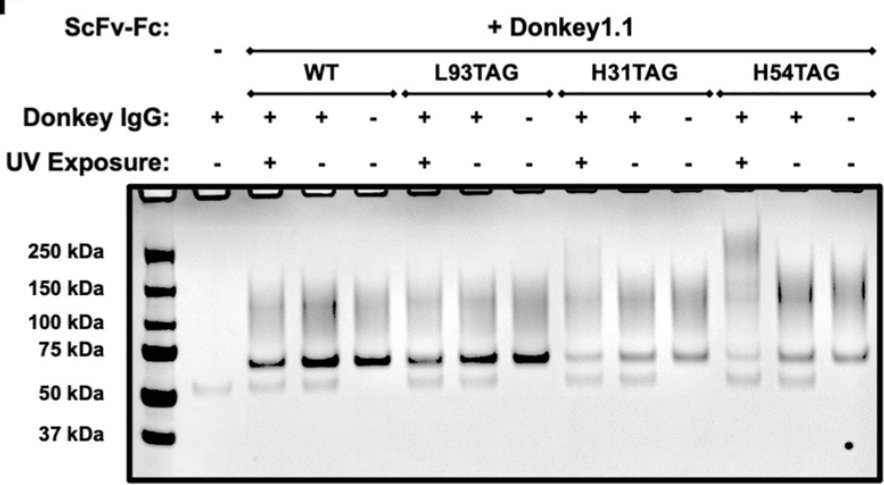


Figure 6. Evaluation of photo-crosslinking on the yeast surface and in solution. A) Strategy for binding competition assay to evaluate photo-crosslinking on the yeast surface. B) Flow cytometry dot plots of competition assay: a decrease in biotinylated IgG detection indicates covalent crosslinking in the presence of UV radiation. "Binding Only (Control)" samples represent binding detection with $500 \mathrm{nM}$ biotinylated IgG in the absence of any competition binding or UV radiation. C) Equations to calculate "Binding Fraction Exchanged," a determination of the fraction of IgG bound noncovalently to scFvs on the yeast surface following the competition assay described in A. D) Analysis of "Binding Fraction Exchanged" for Donkey1.1 (left) and Bovine2.2 (right) clones during the binding competition assay in the absence and presence of UV radiation ("Not Photo-crosslinked" and "Photo-crosslinked" conditions, respectively). All data reported here are the result of crosslinking assays performed in technical triplicates. E) SDS-PAGE and Western Blot analysis to evaluate scFv-Fc purity and confirm AzF substitutions. Top: gel stained with coomassie SimplyBlue SafeStain for samples subjected to CuAAC conditions or left untreated. Bottom: Western blot probed for the presence of biotinyted scFv-Fcs following CuAAC with biotin-alkyne (refer to SI Figure 24 for full gel images). F) SDS-PAGE analysis of photo-crosslinking in solution using $100 \mathrm{nM}$ donkey $\lg \mathrm{G}$ and 500 nM scFv-Fc (refer to SI Figure 25 for full gel images).

Figure $6 \mathrm{~B}-6 \mathrm{D}$ depict the results of a series of crosslinking experiments on the yeast surface using AzF substitutions in Donkey1.1 and Bovine2.2 clones. Inspection of twodimensional dot plots of biotinylated lgG binding versus full-length display detection (Figure 6B and SI Figure 22) indicate clear, UV-dependent decreases in antigen binding levels for AzFsubstituted Donkey1.1 H54TAG and Bovine2.2 H54TAG. The UV-irradiated samples exhibit very low levels of biotinylated antigen compared to the controls, suggesting irreversible binding between the AzF-substituted clones and the target IgGs. In contrast, the high biotin detection for control samples not subjected to UV irradiation indicates the exchange of unmodified IgGs with biotinylated IgGs during the secondary incubation.

To further investigate the effects of different ncAA incorporation sites on crosslinking, we calculated the fraction of biotinylated $\lg$ exchanged as a measure of photo-crosslinking efficiency (Figure 6C and 6D). Here, the single-step biotinylated IgG binding (control) samples were assumed to exhibit maximum levels of antigen binding, with crosslinked and non-crosslinked sample binding calculated as a fraction of the maximum binding. For the WT samples, we generally observed some loss of biotin detection for both photo-crosslinked and non-photocrosslinked conditions after the competition assay. This loss in signal may be attributable to some degree of nonspecific crosslinking even in the absence of AzF or to incomplete exchange of 
unmodified IgG for biotinylated IgG during the competition step of the assay. For the AzFcontaining, photo-crosslinked samples (L93TAG, H31TAG and H54TAG), the biotin detection is generally reduced compared to non-photo-crosslinked samples; the detection of some biotinylated antigen even in UV-irradiated samples suggests that the crosslinking may not be complete. In addition, crosslinking clearly depends on the ncAA incorporation site (Figure 6D and SI Figure 22). While a large fraction of cells exhibit successful crosslinking when displaying clones with AzF substitutions at positions $\mathrm{H} 31$ or $\mathrm{H} 54$, this is not the case for cells displaying scFvs substituted with AzF at the L93 position. This low efficiency may be the result of L93 residing too far from the antigen bound to the CDR-H3-driven antibody paratope to promote efficient crosslinking.

We next investigated whether the trends in photo-crosslinking efficiency observed on the yeast surface would hold when we evaluated crosslinking in solution. To examine this, we prepared scFv-Fc forms of WT and AzF-substituted L93TAG, H31TAG, and H54TAG Donkey1.1 using secretion constructs similar to those described in Van Deventer et al ${ }^{67}$ (with modifications as detailed in Materials and Methods). We confirmed the proper expression of the scFv-Fcs using SDS-PAGE analysis and the presence (or absence) of AzF residues in the purified clones using CuAAC with a biotin-alkyne probe and western blotting (Figure 6E, SI Figures 23 and 24). The protein gel staining patterns of purified samples observed here are extremely similar to those observed in Van Deventer and Kelly et al, with the multiple bands being attributable to glycosylated and aglycosylated forms of the scFv-Fcs. ${ }^{67}$ As shown in Figure 6E, biotin detection on the western blot is only observed for AzF-containing samples that have been subjected to CuAAC, while samples not subjected to CuAAC conditions and all WT samples (irrespective of CuAAC treatments) result in no biotin detection (full gel images reported in SI Figure 24). Thus, CuAAC reactivity of scFv-Fcs expressed in solution is consistent with the results obtained using yeast display: scFv-Fcs retain their ability to selectively undergo CuAAC reactions only when 
prepared from yeast containing a TAG-substituted scFv-Fc and aaRS/tRNA pair and induction with $\mathrm{AzF}$ in the media, thus providing strong evidence for the presence of AzF in these secreted constructs.

With these purified constructs in hand, we performed photo-crosslinking by coincubating the scFv-Fcs with donkey IgG and then exposing them to UV radiation at $365 \mathrm{~nm}$. The resulting samples were then analyzed using SDS-PAGE under reducing conditions to identify potential crosslinking events. Figure $6 \mathrm{~F}$ depicts the analysis of a series of samples subjected to UV irradiation and corresponding controls (full gel images reported in SI Figure 25). Bands larger than both the individual scFv-Fc and the lgG heavy chain band were detected only for the H31TAG and H54TAG photo-crosslinked samples. These observations and the absence of corresponding bands in control samples strongly suggest that the scFv-Fc and IgG have been covalently crosslinked under the conditions used in these experiments. In contrast, there is no detectable band present in samples containing AzF-substituted Donkey1.1 L93TAG. The trends observed in solution therefore appear to be consistent with the trends observed on the yeast surface: crosslinking reactions are readily detectable for clones substituted with $\mathrm{AzF}$ at $\mathrm{H} 31$ and $\mathrm{H} 54$, while AzF substitution at L93 exhibits the lowest apparent amount of crosslinking on the yeast surface and undetectable levels of crosslinking in solution.

These crosslinking experiments demonstrate the preparation and evaluation of covalently crosslinkable antibodies on the yeast surface and in solution. The consistent trends observed between yeast-based and solution-based experiments confirm the utility of conducting initial experiments on the yeast surface prior to initiating more technically demanding characterizations in solution. Moreover, the covalent scFv-antigen linkages formed here are a clear example of the presentation of unique functionality within synthetic antibodies that is not accessible through the side chains of the canonical amino acids. 


\section{Conclusions}

In this work, we investigated the functionality of a minimalist synthetic antibody library and identified strategies for chemically diversifying clones emerging from the library. The singleframework, single-CDR design used in this work is one of the simplest possible diversification schemes, and may be the simplest antibody library reported in yeast display format (at least to our knowledge). Despite such constrained diversity, we isolated moderate to high affinity antibody binders against several animal lgGs using high throughput screening. The affinities of these clones are comparable to the affinities of clones isolated from minimalist phage-based synthetic antibody libraries. ${ }^{25-27}$ Several clones we isolated readily discriminate between donkey, bovine, and rabbit IgGs, indicating that the sequence of a single CDR loop is sufficient to enable identification of specific clones.

Limiting the location of antibody diversity to CDR-H3 enabled us to systematically explore the effects of installing chemical groups within additional CDRs of several antibody clones. Using previously characterized aminoacyl-tRNA synthetase/tRNA pairs, ${ }^{63}$ we identified multiple clones that retained binding function following the incorporation of structurally diverse noncanonical amino acids at locations within CDR-H1, CDR-H2, and CDR-L3. Reductions in antigen binding could be partially explained by the reduction in display levels accompanying the stop codon suppression systems (translation efficiencies at stop codons were less efficient than wild-type translation efficiency under the conditions used here). Titrations of several clones indicated that ncAA substitutions tend to decrease apparent antigen binding affinity, with reductions in affinity ranging from approximately 4 -fold to greater than an order of magnitude (Table 3 ). This observation suggests that ncAA substitutions outside of CDR-H3 impact binding affinity even though these antibody regions were not included in the diversification scheme. However, because changes in display level also change clone avidity on the yeast surface, ${ }^{112-113}$ we cannot rule out 
potential avidity effects without further studies. Despite these caveats, the presentation of ncAAs within these simple synthetic antibody clones is reasonably well-tolerated.

Successful yeast display of functional clones containing ncAAs near antibody binding interfaces enabled us to investigate both bioorthogonal chemistries and photo-crosslinking for exploiting these "chemically diversified" clones. The versatility of the display format supported efficient investigation of each these schemes. Bioorthogonal CuAAC and SPAAC reactions were readily achieved on the yeast surface, in line with previous ncAA-mediated functionalization results from our group and others on yeast ${ }^{62-63,114-115}$ and $E$. coli $i^{36,53,116-117}$ displaying ncAAcontaining proteins. Strikingly, in most substituted clones, installation of bulky probes near the antibody binding interface did not appear to overtly affect binding function. Photo-crosslinking experiments with $p$-azido-L-phenylalanine on the yeast surface strongly suggest site-dependent formation of covalent linkages with target antigens. Importantly, we were able to confirm key observations made on the yeast surface with soluble versions of ncAA-substituted clones. Assays of both CuAAC reactivity and photo-crosslinking in solution yielded results consistent with our initial yeast display-based characterizations. The confirmation that ncAA-mediated properties are retained when displayed clones are converted to soluble form is critical, indicating that yeastbased evaluations of ncAA-containing proteins are predictive of solution behavior.

Overall, our findings elucidate new routes to leveraging an expanded chemical dimension in engineering the properties of synthetic antibodies. The antibody diversification strategy used here supports chemical diversification within many portions of the antibody variable domain; we expect this approach to be transferrable to other binding protein scaffolds. ${ }^{75,118-122}$ We note that the careful introduction of additional antibody diversity, such as using multiple frameworks, ${ }^{22,25,65}$ may enhance antibody library performance while still supporting rapid chemical diversification. In any case, the reactivities accessed through the clickable and photo-active ncAAs used in this work would be challenging or impossible to access using canonical amino acids alone. Moreover, 
the use of yeast display streamlines the evaluation of ncAA-substituted clones in comparison to soluble antibody production and characterization. The binding and reactive properties of clones identified in this study highlight the potentially broad scope of antibody modifications achievable with additional chemical functionalization. However, we recognize that there are numerous effective bioconjugation strategies that may be applied to prepare chemically diversified antibodies, including thiol modification strategies, ${ }^{47,}{ }^{49}$ enzymatic modification strategies, ${ }^{48}$ or emerging conjugation reactions at other canonical amino acids. ${ }^{52,123}$ In this regard, the simple synthetic antibodies and characterization strategies elucidated in this work could facilitate sideby-side evaluations of multiple chemical modification strategies. Finally, while many routes to prepare chemically modified peptides in display formats are now available ${ }^{29,35-36,124-131}$ very few analogous approaches for the chemical diversification of proteins have been described. ${ }^{53,60}$ The efficient preparation and chemical diversification of antibodies on the yeast surface opens up new possibilities for discovering "drug-like" protein leads in high throughput.

\section{Materials and Methods}

Materials. The Saccharomyces cerevisiae strain RJY100 was constructed as described previously. ${ }^{67}$ The ncAA $p$-azido-L-phenylalanine was purchased from Chem-Impex International, Inc., p-propargyloxyphenylalanine and $\mathrm{H}$-L-Lys(EO-N3)-OH* $\mathrm{HCl}$ were purchased from Iris Biotech $\mathrm{GmbH}$ and $p$-azidomethyl-L-phenylalanine purchased from SynChem, Inc. All restriction enzymes used for cloning were from New England Biolabs. Primary and secondary antibodies used for flow cytometry labeling were purchased from Gallus Immunotech (chicken anti-cMyc), BioLegend (mouse anti-HA), Thermo Fisher Scientific (goat anti-chicken Alexa Fluor 647, goat anti-mouse Alexa Fluor 488, streptavidin Alexa Fluor 488 and rabbit anti-donkey DyLight 488), Jackson ImmunoResearch (goat anti-bovine Alexa Fluor 488) and eBioscience (anti-biotin PE). All PCR amplifications were performed with New England Biolabs Q5 DNA polymerase. Synthetic oligonucleotides for cloning and sequencing were purchased from Eurofins Genomics or IDT DNA Technologies. gBlocks encoding the heavy and light chain frameworks used for library construction were synthesized by IDT DNA Technologies. All sequencing in this work was performed by Eurofins Genomics (Louisville, KY) or Quintara Biosciences (Cambridge, MA). Mix and Go! kits from Zymo Research were used to prepare competent E. coli. Epoch Life Science GenCatch $^{\text {TM }}$ Plasmid DNA Mini-Prep Kits and Macherey-Nagel NucleoBond Xtra Maxi Plus EF kits were used to isolate plasmid DNA from E. coli. Frozen-EZ Yeast Transformation II kits and Zymoprep DNA isolation kit from Zymo Research were used to prepare and transform competent yeast, and isolate plasmid DNA from yeast, respectively. Dynabeads ${ }^{\mathrm{TM}}$ Biotin Binder from Thermo 
Fisher Scientific were used for magnetic bead sorting. Penicillin-Streptomycin 100X from Corning was used during propagation of yeast cultures. Chrompure Donkey lgG whole molecule, Chrompure Bovine IgG whole molecule and Chrompure Rabbit IgG whole molecule were purchased in unconjugated forms and in Biotin-SP conjugated forms from Jackson ImmunoResearch. EZ-Link ${ }^{\text {TM }}$ NHS-LC-Biotin, Zeba ${ }^{\text {TM }}$ Spin Desalting Columns, 7K MWCO, 0.5 $\mathrm{mL}$ and Pierce ${ }^{\mathrm{TM}}$ Biotin Quantitation Kits were purchased from Thermo Scientific and used for antigen biotinylation. Biotin-PEG ${ }_{3}-$ Azide, Biotin-PEG - -Alkyne, Biotin-PEG 4 -DBCO and THPTA were purchased from Click Chemistry Tools for use in CuAAC and SPAAC reactions. (+)-Sodium L-ascorbate, Aminoguanidine hydrochloride, copper sulfate pentahydrate, EDTA and DMSO were purchased from Sigma Aldrich for use in CuAAC reactions. 4-12\% Bis-Tris mini gels, SimplyBlue Safestain and iBlot ${ }^{\mathrm{TM}}$ Transfer Stacks were purchased from Thermo Scientific and used for SDSPAGE and Western Blot analysis.

Media preparation. LB, SD-CAA, SG-CAA, and YPG media were prepared as described in Van Deventer et al. ${ }^{67,72}$ SD-SCAA and SG-SCAA were prepared as described in Stieglitz et al. ${ }^{63}$ All SD-SCAA and SG-SCAA media used in this study was prepared without tryptophan, leucine and uracil (-TRP, -LEU, and -URA).

Noncanonical amino acid liquid stock preparation. For all experiments, stocks of noncanonical amino acids were prepared at $50 \mathrm{mM}$ concentration of the L-isomer. NcAAs were dissolved in approximately $90 \%$ of the final desired volume of deionized water and vortexed thoroughly. Either $6 \mathrm{~N}$ or $1 \mathrm{~N}$ sodium hydroxide was used to ensure complete dissolving when needed. Once the stocks were determined to be fully dissolved, deionized water was added to the final volume needed to reach a concentration of $50 \mathrm{mM} \mathrm{L}$-isomer and the stocks were sterile filtered using a $2 \mu \mathrm{m}$ syringe filter. Stocks were prepared fresh prior to each induction.

Yeast transformations, propagation and induction. For display constructs encoding proteins containing only canonical amino acids, display plasmids (pCTCON2 backbone, TRP marker) were transformed into Zymo competent RJY100 cells, plated on SD-CAA (-TRP -URA) solid media and allowed to grow for $2-3$ days at $30{ }^{\circ} \mathrm{C}$, until colonies appeared. For display constructs encoding a TAG codon at L93, H31 and H54 positions, display constructs (pCTCON2 backbone, TRP marker) were transformed alongside aaRS/tRNA suppressor plasmids (pRS315 backbone for LeuOmeRS and LeuRS BH5 T252A, LEU marker; kanamycin resistance modified pRS315 backbone for AcFRS, LEU marker) using Zymo competent RJY100 cells, plated on SD-SCAA TRP - LEU -URA solid media and allowed to grow for 2-3 days at $30^{\circ} \mathrm{C}$, until colonies appeared. The details of display plasmid and aaRS/tRNA plasmid combinations used in this study are listed in SI table 7. For propagation, colonies were inoculated in $2-5 \mathrm{~mL}$ of the respective liquid media supplemented with penicillin-streptomycin at 1:100 dilution (+ Pen/Strep) and left to grow at 30 ${ }^{\circ} \mathrm{C}$ with shaking at 250-300 RPM until saturation (2-3 days). Saturated cultures were stored at 4 ${ }^{\circ} \mathrm{C}$ for future propagation. To propagate, $100-500 \mu \mathrm{L}$ of cells from the saturated culture were pelleted and resuspended to an OD600 of 0.5-1 in $5 \mathrm{~mL}$ of fresh liquid media + Pen/Strep and grown overnight at $30{ }^{\circ} \mathrm{C}$ with shaking at 250-300 RPM. Once saturated, cultures were diluted to an OD600 of 1 and allowed to grow to mid-log phase (OD600 $=2-5 ; 4-8$ hours) and then induced by pelleting and resuspending in the corresponding SG-SCAA media + Pen/Strep to an OD of 1. SG-CAA was used for cultures containing reporter construct plasmids only, SG-SCAA -TRP LEU -URA was used for cultures containing reporter construct plasmids as well as aaRS/tRNA 
suppression machinery. To facilitate ncAA incorporation, induction media for cultures with TAGmutated reporter plasmids were supplemented with ncAAs to a final concentration of $1 \mathrm{mM}$ of the $\mathrm{L}$-isomer (ncAAs used in different experiments are specified in later sections below). Cells were grown in the induction media at $20^{\circ} \mathrm{C}$ with shaking at 250-300 RPM for 15-18 hours before further experimentation.

CDR-H3 library construction and sequence characterization. Oligonucleotides were used to amplify the scFv region, which was split into two regions. The gBlock encoding the first region consisted of the IGKV1-39 light chain and the JK4 J-segment and was amplified with a pCTCON2 overlap region using the primers SynAmpFwd and SidLinkRev (SI Table 3). For the case of the light chain, a DNA sequence encoding the entire IGKV1-39 amino acid sequence was used followed by a sequence encoding the "TFGGGTKVEIK" sequence from JK4. The peptide linker connecting the light and heavy chain variable regions was identical to the linker sequence used in Van Deventer et al $^{67}$ and encoded within the SidLinkRev and SidLinkFwd primers. The gBlock encoding the second region contained the sequence for the IGHV3-23 VH region and was amplified with SidLinkFwd and the CDR9-17Rev primers. The CDR9-17Rev primers varied in length according to the number of designed "XYZ" codons encoded in each primer (labeled as the reverse complement $z^{\prime} y^{\prime} x$ ' in the primer sequence). Additionally, the CDR9-17Rev primers contained an overhang for the first part of the $\mathrm{JH} 4 \mathrm{~J}$-segment sequence. The rest of the $\mathrm{JH} 4 \mathrm{~J}$ segment and the pCTCON2 overlap region were incorporated using SynAmpRev. PCR amplifications of each CDR length were kept separate throughout molecular cloning and yeast electroporation.

The pCTCON2 yeast display vector was linearized based on the method described by Van Deventer and Wittrup 2014. ${ }^{72}$ Approximately $30 \mu \mathrm{g}$ of vector was digested overnight at $37^{\circ} \mathrm{C}$ with $9 \mu \mathrm{L}$ Sall, $30 \mu \mathrm{L} 10 \times$ CutSmart Buffer, and $250 \mu \mathrm{L}$ deionized water. The next day, $9 \mu \mathrm{L}$ each of BamHI and Nhel were added to the reaction and digested overnight at $37^{\circ} \mathrm{C}$. The next morning, $1.5 \mu \mathrm{L}$ of each enzyme were added to the mix for 1 hour before gel purification. $2 \mu \mathrm{g}$ of each gelpurified PCR fragment and $1 \mathrm{\mu g}$ of digested vector mixture were assembled by homologous recombination in electrocompetent RJY100 using previously described methods. ${ }^{72}$ PCR fragments of each CDR-H3 length were electroporated in yeast separately to create loop lengthbased sublibraries. Transformed yeast cultures for each loop length were recovered in SD-CAA (-TRP -URA) + Pen/Strep. Small portions of transformed cells were plated on SD-CAA plates and counted to determine the size of each sublibrary (Table 1). To cryogenically preserve the sublibraries, cultures were expanded in SD-CAA + Pen/Strep, pelleted, resuspended in 15\% glycerol and stored in $2 \mathrm{~mL}$ aliquots each containing $10^{10}$ yeast cells. Plasmid DNA was extracted from each sublibrary using the Zymoprep DNA isolation kit (Zymo Research). DNA was then transformed into E. coli and plated on LB solid media containing ampicillin. The 8-12 E. coli colonies per sublibrary were miniprepped and sequenced (Eurofins Genomics) to assess library diversity.

To prepare the full library from the sublibraries, freezer stocks of each sublibrary were thawed at room temperature and a number of cells equal to 20 times the number of transformants were inoculated in $1 \mathrm{~L} \mathrm{SD}-\mathrm{CAA}+\mathrm{Pen} / \mathrm{Strep}$ at $30^{\circ} \mathrm{C}$. These cells were grown to saturation to create the full CDR-H3 library. Once grown to saturation, 10 times the number of transformants were spun down for 5 minutes at 2,000 rcf and $4{ }^{\circ} \mathrm{C}$, resuspended in $8 \mathrm{~L} \mathrm{SD-CAA}+$ Pen/Strep, 
and grown to saturation overnight. The saturated cultures were spun down, resuspended in $15 \%$ glycerol, and stored in $2 \mathrm{~mL}$ aliquots each containing $10^{10}$ yeast cells at $-80^{\circ} \mathrm{C}$.

Antigen biotinylation. Donkey, bovine and rabbit IgG (Jackson Immuno-Research), and TA99 (prepared according to Zhu et al ${ }^{132}$ ) were diluted from stock concentration to $1 \mathrm{mg} / \mathrm{mL}$ in ice cold 1× PBS. 100 mM EZ-Link NHS-LC biotin solution (Thermo Scientific) was prepared in dimethylformamide (Sigma Aldrich) and mixed extensively to dissolve. Biotinylation reactions were carried out by incubating $100 \mu \mathrm{L}$ aliquots of protein with 2-6 fold molar excess of $100 \mathrm{mM}$ EZ-Link NHS-LC biotin solution for IgGs (including TA99) at room temperature for 30 minutes. Reactions were quenched with $10 \mu \mathrm{L}$ of $0.5 \mathrm{M}$ Tris, $0.02 \% \mathrm{NaN}_{3}, \mathrm{pH} 7.4$. Upon completion of the reaction, proteins were desalted twice using Zeba Spin Desalting Columns with 7K MW cutoff according to the manufacturer's specifications (Thermo Scientific, catalog number 89883). The concentration of the resulting protein was measured via the absorbance of $280 \mathrm{~nm}$ light on a Nano Drop One instrument (Thermo Fisher). The extent of biotinylation was estimated using the HABAAvidin Premix according to the manufacturer's protocol (Thermo Scientific, catalog number 28005). Clear flat-bottomed 96 well plates were used to blank samples by shaking at 150 RPM for one minute, reading absorbance values at $500 \mathrm{~nm}$ and repeating with one-minute shaking intervals until readings were consistent within $\pm 0.003 \mathrm{AU}$. Absorbance measurements were similarly recorded after the addition of biotinylated protein until consistent. The extent of biotinylation (moles of biotin per moles of protein) was calculated from the absorbance readings using the online HABA Calculator by Thermo Scientific, based on the Beer Lambert Law (Thermo Scientific, catalog number 28005).

Flow cytometry data collection and analysis. Analytical flow cytometry was performed as described in previous work. ${ }^{63}$ To prepare scFvs for flow cytometry analysis, 2 million freshly induced cells of each sample were removed from the culture, washed three times in PBSA (1x PBS, $\mathrm{pH} 7.4$, with $0.1 \% \mathrm{w} / \mathrm{v} \mathrm{BSA}$ ) and labeled in either $1.7 \mathrm{~mL}$ microcentrifuge tubes or 96 well $\mathrm{V}$-bottom plates for flow cytometry. Primary labeling was carried out at room temperature for 30 minutes on a rotary wheel (for microcentrifuge tubes) or on an orbital shaker at 150 RPM (for 96well plates). Following primary labeling, all subsequent steps were performed on ice or in a centrifuge chilled to $4{ }^{\circ} \mathrm{C}$. Cells were diluted in ice-cold PBSA and washed twice, also with icecold PBSA. Secondary labelling was performed on ice for 15 minutes in the dark. Samples were diluted in and washed once with ice-cold PBSA before final resuspension in PBSA for flow cytometry. The particular labeling reagents and concentrations used for primary and secondary labelling were dependent on the type of experiment and are listed in SI tables 2, 5-6 and 8-9, 11-15; labeling reagents were prepared in PBSA unless otherwise noted. Flow cytometry was performed on an Attune NxT flow cytometer (Life Technologies) in the Tufts University Science and Technology Center and data was processed using FlowJo software. Unless otherwise stated, 10,000 events were collected per sample on the flow cytometer.

To prepare sublibraries for flow cytometry characterizations, a volume of culture containing 20 times the number of transformants of each sublibrary was grown separately in either 100 or $150 \mathrm{~mL}$ of SD-CAA (-TRP -URA) + Pen/Strep at $30{ }^{\circ} \mathrm{C}$ with shaking at $300 \mathrm{RPM}(150 \mathrm{~mL}$ was used to ensure sufficient coverage of sublibrary CDR14, containing $2.3 \times 10^{8}$ transformants). Once grown to saturation, a volume of cells corresponding to 10 times the number of transformants for each sublibrary was spun down (5 minutes at $2,000 \mathrm{rcf}$ at $4{ }^{\circ} \mathrm{C}$ ) and 
resuspended in 100 or $150 \mathrm{~mL}$ of SD-CAA + Pen/Strep; based on number of transformants in the sublibrary. After growing the cultures to saturation again, approximately $500 \mu \mathrm{L}$ of each sublibrary was passaged into $5 \mathrm{~mL}$ of SD-CAA + Pen/Strep and grown to mid-log phase for 4-8 hours at 30 ${ }^{\circ} \mathrm{C}$ with shaking at 250-300 RPM. Additionally, $500 \mu \mathrm{L}$ of the full CDR-H3 library was passaged into $5 \mathrm{~mL}$ of SD-CAA + Pen/Strep and grown for $4-8$ hours at $30^{\circ} \mathrm{C}$ with shaking (250-300 RPM). For induction, cultures were spun down and resuspended at an OD600 of 1 in SG-CAA + Pen/Strep and grown for approximately 16 hours at $20^{\circ} \mathrm{C}$ with shaking (250-300 RPM).

Data analysis was performed using FlowJo and Microsoft Excel following these general steps. The data was 1) gated to isolate the single cell populations, 2) gated to isolate induced populations (full-length display events with positive c-Myc detection), 3) median fluorescence intensity (MFI) data was retrieved for c-Myc detection of induced populations and uninduced populations (truncated or no protein display), 4) MFI data of induced and uninduced populations was retrieved for other parameters being detected, and 5) background correction was performed by subtracting MFI values of uninduced cell populations from MFI values of corresponding induced populations. Additionally, quadrants were drawn on dot plots based on the putatively uninduced populations of cells in order to identify positive and negative populations. In most cases, quadrants prepared in this way result in c-Myc-positive events in Q2 and Q3, events positive for a second parameter in Q1 and Q2, and background level detection of each parameter in Q4 (corresponding to uninduced or unlabeled populations).

Truncation analysis samples were gated for 1) the induced full-length population and 2) the induced truncated populations. The full-length induced population was defined as exhibiting fluorescence indicating both $\mathrm{HA}$ and c-Myc detection whereas the truncated population was defined as exhibiting fluorescence indicating only HA detection. To obtain the percentage of truncated clones, the percent of the truncated induced population was divided by the total percent of induced population for each CDR sublibrary and the full CDR-H3 library.

Library sorting. Two aliquots of the full, frozen CDR-H3 library were thawed at room temperature and inoculated into a $1 \mathrm{~L}$ SD-CAA (-TRP -URA) + Pen/Strep culture. The culture was grown to saturation at $30{ }^{\circ} \mathrm{C}$ with shaking (250-300 RPM). The library was spun down and resuspended at an OD600 of 1 and grown again to saturation. The remainder of the culture was placed at $4{ }^{\circ} \mathrm{C}$ as a short-term stock. Alternatively, stocks saved at $4{ }^{\circ} \mathrm{C}$ were pelleted and resuspended to an OD600 of 1 in $1 \mathrm{~L}$ of fresh SD-CAA + Pen/Strep and grown to saturation with shaking (250-300 RPM). Following saturation, the cultures were pelleted and resuspended to an OD600 of 1 in fresh media and grown until mid-log phase (OD600 between 2-5; 4-8 hours). Cells were pelleted (5 minutes at 2,000 rcf and $4{ }^{\circ} \mathrm{C}$ ) and resuspended to an OD600 of 1 in SG-CAA + Pen/Strep induction media and grown at $20^{\circ} \mathrm{C}$ for at least 16 hours with shaking (250-300 RPM) before sorting.

150-250 $\mu \mathrm{L}$ of Dynabeads (Life Technologies) were prepared by washing three times in $1 \mathrm{~mL}$ ice-cold PBSA. Washes were done by incubation on a DynaMag2 for 2 minutes followed by aspiration of supernatant. After washing, the beads were resuspended to the original 150-250 $\mu \mathrm{L}$ volume in ice-cold PBSA. Beads were aliquoted in $50 \mu \mathrm{L}$ volumes and left alone or incubated with biotinylated TA99 (33 pmol antigen $/ 10 \mu \mathrm{L}$ beads) or $5.3 \mu \mathrm{L}$ of $0.01 \mathrm{mg} / \mathrm{mL}$ D-biotin stock solution on a rotary wheel at $4{ }^{\circ} \mathrm{C}$ for $2-16$ hours. 
For the first round of negative sorting, $1.5 \times 10^{10}$ induced cells were pelleted, washed and resuspended in $5 \mathrm{~mL}$ ice-cold PBSA. For each sort, prepared beads were washed and added to the cells, then placed at $4{ }^{\circ} \mathrm{C}$ on a rotary wheel for 2 hours. After this incubation, the cells were distributed evenly into five $1.7 \mathrm{~mL}$ microcentrifuge tubes and placed on the DynaMag for 5 minutes. After this time, the supernatants were transferred to a new conical tube. This was repeated for each negative sort against the beads, TA99, and biotin. After the final sort, the remaining cells were rescued in $1 \mathrm{~L} \mathrm{SD-CAA}+$ Pen/Strep for overnight growth until saturation. Then, cells were induced, beads were prepared, and sorts were performed a second time exactly as stated above.

After the second negative sort, cells were rescued overnight and induced as stated above. After overnight induction, cells were pelleted, washed, and resuspended in $5 \mathrm{~mL}$ PBSA as stated above, with beads also washed as stated above. Beads were prepared for positive enrichments by incubating them with either biotinylated donkey, bovine, or rabbit lgG (33 pmol antigen/10 $\mu \mathrm{L}$ beads) on a rotary wheel at $4{ }^{\circ} \mathrm{C}$ for $2-16$ hours. $50 \mu \mathrm{L}$ of antigen-coated beads were added to the suspension culture and placed on a rotary wheel at $4{ }^{\circ} \mathrm{C}$ for 2 hours. Then the cells were incubated on the DynaMag for 5 minutes and the supernatant was transferred to new tube on the DynaMag to ensure no loss of beads. Remaining beads were washed once in $1 \mathrm{~mL}$ ice-cold PBSA and supernatant was discarded after 2 minutes of incubation. Beads and attached cells were rescued in $5 \mathrm{~mL}$ SD-CAA + Pen/Strep at $30^{\circ} \mathrm{C}$ overnight. The next morning, cells were passaged into $50 \mathrm{~mL}$ SD-CAA + Pen/Strep and grown until about OD 3, when cells were pelleted and resuspended in $1 \mathrm{~mL}$ induction media. Cells were incubated on the DynaMag to remove beads and then grown in $50 \mathrm{~mL}$ induction media for overnight growth at $20^{\circ} \mathrm{C}$ to prepare for the fourth round of sorting.

For the fourth round of sorting, $10 \mu \mathrm{L}$ Dynabeads were prepared with the biotinylated antigens needed for negative and positive selections. After overnight growth, cells were pelleted, washed and resuspended in $1 \mathrm{~mL}$ ice-cold PBSA. Cells were subjected to depletions against the Dynabeads, biotin, and TA99 by incubating the cells with beads for 2 hours on a rotary wheel at $4{ }^{\circ} \mathrm{C}$. Cells were then placed on the DynaMag for 5 minutes and supernatants were transferred to a new conical tube for the next sort. For the final positive sort on IgG, the cells were incubated on the DynaMag for 5 minutes and supernatant was removed. The supernatant was transferred to new tube on the DynaMag to ensure no loss of beads. Remaining beads were washed once in $1 \mathrm{~mL}$ ice-cold PBSA and supernatant was discarded after 2 minutes of incubation. Cells were rescued in $5 \mathrm{~mL}$ SD-CAA + Pen/Strep and grown overnight. This process was repeated two or three times.

Following bead-based enrichments, each of these populations were sorted using fluorescence-activated cell sorting (FACS) performed at the Tufts Laser Cytometry Core on a BD FACSAria following four (donkey $\lg G$ ), three to four (bovine $\operatorname{lgG}$ ) or four (rabbit $\lg G$ ) rounds of bead sorting. To prepare samples for sorting, $2 \times 10^{7}$ cells from each population were pelleted and resuspended in PBSA. During primary labeling, cells were labeled with chicken anti-cMyc at a dilution of 1:250 in PBSA and $50 \mathrm{nM}$ biotinylated antigen. During secondary labeling, cells were labeled with goat anti-chicken Alexa Fluor 647 at a dilution of 1:500 in PBSA and anti-biotin PE added to a dilution of 1:500 in PBSA. For each sort, approximately 50,000 to 1 million events were sorted by FACS and approximately 300-8,000 c-Myc-positive, antigen binding-positive events 
were collected. Sorted cells were rescued in $3 \mathrm{~mL} \mathrm{SD-CAA}+$ Pen/Strep at $30^{\circ} \mathrm{C}$ with shaking at 300 RPM; populations were grown to saturation prior to subsequent evaluations.

Analytical flow cytometry was used to evaluate the binding properties of enriched populations. Cells from each population were induced and prepared for flow cytometry as described in the section, "Flow cytometry data collection and analysis". Briefly, for each round of sorting after the first round of positive enrichment, induced cells were evaluated for binding to the desired antigen and streptavidin by flow cytometry. Cells were subjected to the same wash conditions as in "Flow cytometry data collection and analysis". Samples were labeled with $50 \mathrm{nM}$ biotinylated antigen and chicken anti-cMyc at a 1:250 dilution in PBSA. For secondary labeling, cells were labeled with goat anti-chicken Alexa Fluor 647 and either streptavidin Alexa Fluor 488 or anti-biotin PE at a 1:500 dilution.

Binder characterizations. Plasmid DNA was isolated from enriched populations sorted against donkey, bovine and rabbit lgG and transformed into $E$. coli to prepare single clones for sequence analysis. 9-12 clones were sequenced from each of the sorted donkey, bovine and rabbit $\lg G$ populations. Unique clones were transformed into RJY100, plated on solid SD-CAA (-TRP -URA) media, and grown at $30^{\circ} \mathrm{C}$ until colonies appeared (2-3 days). Colonies were inoculated in $5 \mathrm{~mL}$ of SD-CAA + Pen/Strep and allowed to grow to saturation at $30^{\circ} \mathrm{C}$ with shaking (250-300 RPM) for 2-3 days. Alternatively, $100-200 \mu \mathrm{L}$ of saturated culture stored at $4{ }^{\circ} \mathrm{C}$ was inoculated in $5 \mathrm{~mL}$ fresh media and allowed to grow to saturation overnight. Following saturation, the cultures were diluted to an OD600 of 1 in fresh SD-CAA + Pen/Strep and grown at $30^{\circ} \mathrm{C}$ until mid-log phase (OD 2-5; 4-8 hours). Cells were pelleted (5 minutes at 2,000 rcf and $4{ }^{\circ} \mathrm{C}$ ) and resuspended to an OD600 of 1 in SG-CAA. Cells were prepared for flow cytometry analysis as described in the section "Flow cytometry data collection and analysis," labelling for biotinylated lgG (using either $50 \mathrm{nM}$ or $250 \mathrm{nM}$ biotinylated antigen) and c-Myc according to SI Table 2 (secondary antibodies were used in a 1:500 dilution).

Biotinylated antigen competition. Biotin competition assays were used to determine if clones preferentially recognize biotinylated antigen over unconjugated antigen. ${ }^{76}$ To conduct these experiments, induced RJY100 cells displaying unique scFvs isolated from the CDR-H3 library (SI Table 5) were washed three times in PBSA, and first labeled with 1 or $5 \mu \mathrm{M}$ unconjugated antigen and chicken anti-cMyc at a 1:500 dilution in PBSA. Cells were incubated for 30 minutes at room temperature on an orbital shaker at 150 RPM. After incubation, the plate was kept on ice or at 4 ${ }^{\circ} \mathrm{C}$ for the remainder of the experiment. Biotinylated antigen was added to a final concentration of 50 or $250 \mathrm{nM}$ (for the initial unmodified antigen concentrations of 1 or $5 \mu \mathrm{M}$, respectively). Cells were mixed and incubated on an orbital shaker at 150 RPM for 15 minutes at $4{ }^{\circ} \mathrm{C}$. After the incubation, cells were diluted in $200 \mu \mathrm{L}$ ice cold PBSA and washed three times at $4{ }^{\circ} \mathrm{C}$. Secondary labeling and subsequent data collected was performed as outlined in "Flow cytometry data collection and analysis" and SI Table 2 and 5.

Introduction of TAG codons into scFvs. Primers encoding a TAG codon at L93, H31 or H54 positions (SI Table 3) were used to introduce mutations to the Donkey1.1, Bovine2.1, Bovine2.2 and Rabbit1.1 scFvs. Two DNA segments were amplified via PCR for each scFv, one with the pCTCON2Fwd + \#\#\#AGRev and one with \#\#\#AGFwd + pCTCON2Rev primers (\#\#\# refers to the mutation sites $\mathrm{L} 93, \mathrm{H} 31$ or $\mathrm{H} 54$ ) to introduce the mutation at the desired position. Gel 
electrophoresis was performed on the PCR products to extract DNA fragments of the expected insert size, and then recombined with a pCTCON2 vector digested at the Nhel and BamHI restriction sites using Gibson Assembly. The assemblies were transformed into chemically competent $E$. coli $\mathrm{DH} 5 \alpha \mathrm{Z1}$, plated on LB plates containing ampicillin and grown at $37^{\circ} \mathrm{C}$ overnight. Resulting colonies were picked and grown to saturation in $5 \mathrm{~mL}$ LB liquid media containing ampicillin. Plasmid DNA was then isolated from these cultures using GenCatch Plasmid DNA Mini-Prep kits (Epoch Life Sciences). The cloned plasmids were digested at the Nhel and BamHI sites to confirm the presence of correctly sized inserts by performing gel electrophoresis. Finally, the plasmids were sequence verified from Quintara Biosciences.

RRE and MMF calculations. For relative readthrough efficiency (RRE) and maximum misincorporation frequency (MMF) calculations, all display plasmids (with or without TAG codon mutations) were transformed along with aaRS/tRNA suppressor plasmids using Zymo-competent RJY100 cells and plated on SD-SCAA -TRP -LEU -URA solid media. Colonies were grown in SD-SCAA -TRP -LEU -URA + Pen/Strep liquid media as described above, and induced in SGSCAA -TRP - LEU -URA + Pen/Strep both in the presence of $1 \mathrm{mM}$ ncAA as well as in the absence of ncAA. Three separate transformants per antibody clone + aaRS/tRNA combination were used to generate the data for RRE and MMF measurements. Data analysis was performed as described previously by Stieglitz et al ${ }^{63}$ and Potts et al. ${ }^{64}$

Binding affinity $\left(\mathrm{K}_{\mathrm{D}}\right)$ data collection and analysis. 1.5 million freshly induced RJY100 cells displaying scFvs were aliquoted in $1.7 \mathrm{~mL}$ microcentrifuge tubes, pelleted, washed three times with $1 \times$ PBSA, and then resuspended in $1 \mathrm{~mL}$ PBSA. $300 \mu \mathrm{L}$ of the washed cells were then combined with $0.3 \mu \mathrm{L}$ of chicken anti-cMyc, vortexing gently to mix. To prepare for binding titration, donkey, bovine or rabbit IgG was added to wells of 96 -well V-bottom plates starting at $1 \mu \mathrm{M}$ with seven subsequent 4-fold dilutions and a final PBSA blank (no lgG). To each of these 9 lgG concentrations, $10 \mu \mathrm{L}$ of the cells incubated with chicken anti-cMyc were added, resulting in approximately 15,000 cells per sample, and incubated on the orbital shaker at 150 RPM at room temperature overnight (final chicken anti-cMyc dilution of 1:1000). Wild-type Donkey1.1 and its ncAA-containing variants (at L93, H31 and H54 positions) were added to wells containing different concentrations of biotinylated donkey lgG; wild-type Bovine2.2 and its ncAA-containing variants, wild-type Bovine2.1 and wild-type Bovine2.3 were added to biotinylated bovine IgG; and finally, wild-type Rabbit1.1 was added to biotinylated rabbit IgG. After the overnight incubation with chicken anti-cMyc, samples were diluted in and washed 3-5 times with ice-cold PBSA. Secondary labeling and flow cytometry data collection was performed as described for scFvs in the "Flow cytometry data collection and analysis" above, with the exception that 3,000 events were collected per sample instead of the usual 10,000 events. Reagents used for secondary labeling are listed in SI table 11. All titrations were done in technical triplicates.

Data analysis to obtain binding affinities $\left(\mathrm{K}_{\mathrm{D}}\right)$ were done using steps similar to those outlined in the "Flow cytometry data collection and analysis" section above, with a few modifications. Samples were gated to isolate single cells, and gated on c-Myc detection levels to isolate positive (induced) and negative (uninduced) populations. MFI data for biotinylated antigen detection of induced populations and uninduced populations was retrieved. Background correction was performed by subtracting biotinylated antigen detection MFI values of uninduced cell populations from MFI values of corresponding induced populations. On GraphPad Prism, the 
background corrected data for biotinylated antigen detection were normalized for binding (by converted MFI values to ratios between the highest IgG concentration and all subsequent lower concentrations) and then, using the "Receptor binding - Saturation binding" model and "One site -- Specific binding" equation, the data was fitted to a curve and $K_{D}$ estimated including standard error and $95 \%$ confidence interval.

Click chemistry on the yeast surface. Copper-catalyzed azide-alkyne cycloaddition (CuAAC) reactions were performed as described previously using $100 \mu \mathrm{M}$ concentration of biotin-alkyne or biotin-azide probes. ${ }^{62-63}$ Strain-promoted azide-alkyne cycloaddition (SPAAC) reactions were carried out essentially as previously described with minor modifications. ${ }^{62}$ Induced cells were pelleted, washed with PBSA pH 7.4 three times, and resuspended in $248.75 \mu \mathrm{L}$ ice-cold PBS $\mathrm{pH}$ 7.4. 1.25 $\mu \mathrm{L} 2 \mathrm{mM} \mathrm{DBCO}-\mathrm{PEG}_{4}$-Biotin (Click Chemistry Tools), prepared in DMSO, was added to each sample and vortexed briefly. SPAAC reactions were allowed to run for 2 hours at $4{ }^{\circ} \mathrm{C}$ on a rotary wheel, after which the samples were diluted in PBSA, pelleted, and then washed three times with ice-cold PBSA. Following the click chemistry reactions (both CuAAC and SPAAC), each sample was split into two and prepared for flow cytometry analysis. One set of samples were labeled to detect the biotin probes as a measure of click chemistry reactions; the other set labeled to detect IgG treatment after click chemistry reactions. For the sample set detecting lgG binding, Donkey1.1 clones were treated with $200 \mathrm{nM}$ donkey lgG, and Bovine2.2 clones were treated with $200 \mathrm{nM}$ bovine IgG during primary labeling. Both sample sets were also labeled to detect c-Myc (full-length display). Primary and secondary labeling conditions are outlined in the "Flow cytometry data collection and analysis" section above for scFvs, and antibodies used for labeling are reported in SI Table 14. All CuAAC and SPAAC reactions and subsequent labeling experiments were done in technical triplicates.

Evaluation of click chemistry flow cytometry data was performed using FlowJo and Microsoft Excel with steps similar to those described above. Briefly, we 1) gated samples to isolate single cell, c-Myc positive populations, 2) retrieved MFI data for c-Myc detection of induced populations, 3) retrieved MFI data for biotin detection for click chemistry analysis of induced populations and uninduced populations, 4) retrieved MFI data for IgG detection for binding analysis of cMyc-positive (induced) populations and uninduced populations, and 5) performed background correction by subtracting biotin or IgG detection MFI values of uninduced cell populations from MFI values of corresponding induced populations. Background correct IgG detection MFI values of the technical triplicates were averaged and used to evaluate the observed binding of the samples. Standard deviations were also calculated using the STDEV.S function on Microsoft Excel and used as the measure of error.

Photo-crosslinking on the yeast surface. Two million freshly induced RJY100 cells displaying Donkey1.1 or Bovine2.2 scFvs and their ncAA containing variants (see SI Table 15) were transferred to a 96-well V-bottom plate, pelleted, washed three times with PBS, and then subjected to three types of sample preparation: photo-crosslinked, non-photo-crosslinked controls, and binding controls. For the photo-crosslinked sample set, samples were resuspended in $50 \mu \mathrm{L} 200 \mathrm{nM}$ IgG (either donkey or bovine) and incubated for 30 minutes at room temperature with orbital shaking at 150 RPM. Next, samples were transferred to a 96-well clear flat-bottomed plate lined with aluminum foil, shiny side facing the plate (referred to as the "irradiation plate" below), and irradiated without the plate lid using low intensity $365 \mathrm{~nm}$ UV radiation from a $8 \mathrm{~W}$ 
hand-held UV lamp positioned 1-2 inches from the samples (Spectroline EN-180) for 6 hours on ice, resuspending once every hour. The samples were then diluted in $50 \mu \mathrm{L}$ ice-cold PBS, resuspended extensively and transferred to a new 96-well V-bottomed plate. To maximize the recovery of cells after UV exposure, the wells of the irradiation plate were rinsed three more times with $50 \mu \mathrm{L}$ ice-cold PBS, each time resuspending thoroughly and transferring to the corresponding well on the 96-well plate. The samples were pelleted and washed three times with ice-cold PBS. Following this, samples were resuspended in $50 \mu \mathrm{L} 500 \mathrm{nM}$ biotinylated IgG (donkey lgG for Donkey1.1 clones and bovine IgG for Bovine2.2 clones) containing chicken anti-cMyc and incubated within the range of 2 hours to overnight at room temperature with orbital shaking at 150 RPM. Samples were then diluted in and washed twice with ice-cold PBSA. Subsequent steps starting from secondary labeling were performed according to the "Flow cytometry data collection and analysis" section above. For the non-photo-crosslinked controls, samples were first resuspended in $50 \mu \mathrm{L} 200 \mathrm{nM}$ lgG (donkey lgG for Donkey1.1 clones and bovine lgG for Bovine2.2 clones) and incubated for 30 minutes at room temperature with orbital shaking at 150 RPM, after which the samples were left stationary on ice for 6 hours in the dark, resuspending once every hour. Next, the samples were diluted in $200 \mu \mathrm{L}$ ice-cold PBS and washed three times with ice-cold PBS. Following this, samples were resuspended in $50 \mu \mathrm{L} 500 \mathrm{nM}$ biotinylated $\lg \mathrm{G}$ (donkey or bovine as required) containing chicken anti-cMyc and incubated within the range of 2 hours to overnight at room temperature with orbital shaking at 150 RPM and then prepared for flow cytometry as described above and in the "Flow cytometry data collection and analysis" section. For the binding controls, samples were resuspended in $50 \mu \mathrm{L} 500 \mathrm{nM}$ biotinylated lgG (donkey or bovine as required) containing chicken anti-cMyc after the initial washes, incubated within the range of 2 hours to overnight at room temperature with orbital shaking at 150 RPM and then prepared for flow cytometry analysis similarly to the photo-crosslinked and non-photocrosslinked sample sets. Details of labeling conditions are listed in SI Table 15. Photocrosslinking, non-photo-crosslinking and binding control conditions were all tested in technical triplicates.

Data from photo-crosslinking flow cytometry experiments was analyzed using a combination of FlowJo and Microsoft Excel as described earlier, with modifications as detailed here. First, samples were gated to include only single cell and c-Myc positive populations. Second, using the quadrant gating from dot plots, the population count of Q2 (full-length scFvs binding to biotinylated lgG) was divided by population count of Q2 + Q3 (full-length scFvs) to calculate the percentage of cells in which binding was detected (referred to as "apparent percentage binding" below and elsewhere in the manuscript). Last, the fraction binding exchanged was calculated by dividing the apparent percentage binding of each sample by the apparent percentage binding of the control (binding only) sample (see equations in Figure 6C). Values calculated for the fraction binding exchanged of the technical triplicates were averaged and used to evaluate photo-crosslinking; standard deviations were obtained using the STDEV.S function on Microsoft Excel and used as the measure of error.

Production and purification of soluble scFv-Fcs. To facilitate ncAA incorporation, the TAG stop codon in a previously established yeast secretion plasmid, ${ }^{67}$ pCHA-FcSup-TAG, was first converted to a TAA stop codon. Primers used to encode a TAA codon in place of the TAG codon in the pCHA-FcSup-TAG plasmid backbone are listed in SI Table 3. Two DNA segments were 
amplified via PCR, one with the pCHA_TAAFwd1 + pCHA_TAARev1 and one with pCHA_TAAFwd2 + pCHA_TAARev2 primers, and then recombined with the pCHA-FcSup-TAG vector digested at the Xmal and Xhol restriction sites using Gibson Assembly. In the second step, DNA encoding Donkey1.1 WT, L93TAG, H31TAG and H54TAG scFvs was amplified via PCR using the pCHA_scFvFwd and pCHA_scFvRev primers. Each PCR amplified insert was then recombined with the pCHA-FcSup-TAA vector digested at the Nhel and Xmal restriction sites using Gibson Assembly. Quality control steps to isolate PCR products of approximately the correct size and successful Gibson Assembly reactions, as well as general transformation and plasmid isolation steps were performed as described in the "Introduction of TAG codons into scFvs" section. All plasmids were sequence verified with sequencing performed at Quintara Biosciences.

ScFv-Fcs were secreted and purified according to Van Deventer et al, $2015,{ }^{67}$ with the following modifications. For the WT antibody (containing only canonical amino acids), the secretion plasmid ( $\mathrm{pCHA}-\mathrm{FcSup}-\mathrm{TAA}$ backbone, TRP marker) was transformed into Zymo competent RJY100 cells, inoculated and grown in SD-CAA (-TRP -URA) + Pen/Strep, and induced in $100 \mathrm{~mL} \mathrm{YPG} \mathrm{+} \mathrm{Pen/Strep} \mathrm{at} 20^{\circ} \mathrm{C}$ with shaking at 250-300 RPM for 4 days. Following induction, the culture was pelleted for 35 minutes at 3214 rcf, filtered using a $0.2 \mu \mathrm{M}$ filter and $\mathrm{pH}$ adjusted to 7.4 using $10 \times$ PBS. The filtrate was passed twice through a protein A column, following which the scFv-Fc containing resin was washed three times with $10 \mathrm{~mL}$ PBS. The scFv-Fcs were then eluted using $7 \mathrm{~mL} 100 \mathrm{mM}$ glycine, $\mathrm{pH} 3.0$ and immediately neutralized with $0.7 \mathrm{~mL} 1 \mathrm{M}$ Tris, $\mathrm{pH}$ 8.5. The eluant was buffer exchanged using Amicon Ultra-15 centrifugal filter units (30 kDa molecular weight cut-off, Millipore Sigma) into PBS, pH 7.4 and concentrated. Protein concentrations were measured via the absorbance of $280 \mathrm{~nm}$ light on a Nano Drop One instrument (Thermo Fisher). Protein purity was determined via sodium dodecyl sulfatepolyacrylamide gel electrophoresis (SDS-PAGE) without the use of PNGase F (SI Figure 23). For scFv constructs encoding a TAG codon at L93, H31 or H54, secretion plasmids (pCHA-FcSupTAA backbone, TRP marker) were co-transformed with the pRS315-KanRmod-AcRFS aaRS/tRNA suppressor plasmid (LEU marker) using Zymo competent RJY100 cells, inoculated and grown in SD-SCAA -TRP -LEU -URA + Pen/Strep, and induced in 1 L YPG + Pen/Strep + $1 \mathrm{mM} \mathrm{AzF}$ at $20{ }^{\circ} \mathrm{C}$ with shaking at 250-300 RPM for 4 days in the dark. The subsequent purification steps were identical to that of the WT purification, with the additional step of keeping all protein samples putatively containing AzF (supernatant, filtrate, resin and eluate) in the dark. Protein purity was determined via SDS-PAGE (SI Figure 23).

Click chemistry on soluble scFv-Fcs. CuAAC click chemistry reactions were performed using $100 \mu \mathrm{M}$ concentrations of biotin probes based on previous protocols. ${ }^{133}$ In microcentrifuge tubes containing $220 \mu \mathrm{L}$ aliquots of $500 \mathrm{nM}$ scFv-Fc in 1× PBS pH7.4, we added 1) $1.25 \mu \mathrm{L} 20 \mathrm{mM}$ biotin-(PEG) $)_{4}$-alkyne, 2) $3.8 \mu \mathrm{L} \mathrm{CuSO}_{4} / \mathrm{THPTA}$ (1:2 ratio of $20 \mathrm{mM} \mathrm{CuSO}_{4}: 50 \mathrm{mM}$ THPTA), 3) $12.5 \mu \mathrm{L} 100 \mathrm{mM}$ aminoguanidine and 4) $12.5 \mu \mathrm{L} 100 \mathrm{mM}$ sodium ascorbate (in the given order), vortexing briefly in between each addition. The reactions were left to proceed for one hour at room temperature, following which they were quenched using $2 \mu \mathrm{L}$ of $100 \mathrm{mM}$ EDTA. ScFv-Fcs that had undergone reactions with DMSO in place of biotin probes and scFv-Fcs that had not been subjected to click chemistry reactions were used as controls. Western blots were used to analyze click chemistry reactions and verify the presence of AzF in the TAG-mutated scFv-Fcs. SDS- 
PAGE using 4-12\% Bis-Tris gels (Life Technologies) was performed on all samples in duplicate gels. One gel was stained with coomassie SimplyBlue SafeStain (Life Technologies) to confirm the presence of protein. Western blots were performed by transferring the second PAGE gel to a nitrocellulose membrane using an iBlot2 Dry Blotting System (Life Technologies). Transferred membranes were blocked overnight with $5 \% \mathrm{w} / \mathrm{v}$ BSA in TBS $+0.1 \%$ Tween 20 , following which they were probed with streptavidin Alexa Fluor 488 in a 1:1000 dilution for the presence of biotin (Figure 6E and SI Figure 24).

Photo-crosslinking of soluble scFv-Fcs. For photo-crosslinked samples, $25 \mu \mathrm{L}$ of $200 \mathrm{nM}$ donkey lgG was aliquoted to wells of a 96-well clear flat-bottomed plate containing $25 \mu \mathrm{L}$ of $1 \mu \mathrm{M}$ Donkey1.1 WT, L93TAG, H31TAG or H54TAG scFv-Fc (for a final concentration of $100 \mathrm{nM}$

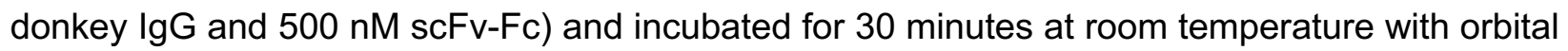
shaking at 150 RPM. Next, the bottom of the plates were lined with aluminum foil, shiny side facing the plate, and samples irradiated without the lid using low intensity $365 \mathrm{~nm}$ UV radiation from a 8 W hand-held UV lamp (Spectroline EN-180) for 2 hours on ice, mixing once every hour during irradiation. For non-photo-crosslinked control samples, $25 \mu \mathrm{L}$ of $200 \mathrm{nM}$ donkey lgG was aliquoted to wells of a 96-well clear flat-bottomed plate containing $25 \mu \mathrm{L}$ of one of the four $1 \mu \mathrm{M}$ scFv-Fcs (for a final concentration of $100 \mathrm{nM}$ donkey lgG and $500 \mathrm{nM} \mathrm{scFv-Fc}$ ) and incubated for 30 minutes at room temperature with orbital shaking at 150 RPM, and then left in the dark for 2 hours on ice, mixing once every hour while on ice. SDS-PAGE using 4-12\% Bis-Tris gels (Life Technologies) was then performed for all samples alongside donkey IgG only and scFv-Fc only controls for comparison (Figure 6F and SI Figure 25). Gels were stained with coomassie SimplyBlue SafeStain (Life Technologies) and imaged using the visible settings on a c400 imaging system (Azure Biosystems).

\section{Associated Content}

\section{Supporting Information}

Supplementary figures provide in depth library characterizations and binding, click chemistry and photo-crosslinking data for additional clones. Supplementary tables provide detailed information on experimental conditions used throughout this work, additional library characterizations, and relative readthrough efficiency and maximum misincorporation frequency data for noncanonical amino acid incorporation. Supplementary methods describe click chemistry time courses, the cloning of the AcFRS suppression machinery, and the cloning of control display constructs.

\section{Author Information}

\section{Corresponding Author}

*James.Van Deventer@tufts.edu

4 Colby Street Room 148, Medford, MA 02155.

\section{Author Contribution}

${ }^{\perp}$ M.I. and ${ }^{\perp}$ H.P.K. contributed equally.

\section{Notes}

The authors declare no competing financial interest. 


\section{Acknowledgments}

Research reported in this publication was supported by the National Cancer Institute of the National Institutes of Health under Award Number R21CA214239, Tufts startup funds, and an award from the Tufts Faculty Research Awards Committee. The content is solely the responsibility of the authors and does not necessarily represent the official views of the National Institutes of Health or Tufts University. The authors also thank Jessica Stieglitz for providing modified suppression plasmids for use in ncAA incorporation experiments.

\section{Abbreviations}

CDR, complementarity determining region

scFv, single-chain variable fragment

FACS, fluorescence-activated cell sorting

aaRS, aminoacyl-tRNA synthetase

OTS, orthogonal translation system

ncAA, noncanonical amino acid (also unnatural amino acid, nonstandard amino acid, non-native amino acid, or non-natural amino acid)

$R R E$, relative readthrough efficiency

MMF, maximum misincorporation frequency

AzF, p-azido-L-phenylalanine

OPG, $p$-propargyloxyphenylalanine

AzMF, $p$-azidomethyl-L-phenylalanine

LysN3, H-L-Lys(EO-N3)-OH

CuAAC, copper-catalyzed azide-alkyne cycloaddition

SPAAC, strain-promoted azide-alkyne cycloaddition

DBCO, dibenzocyclooctyne

DMSO, dimethyl sulfoxide 


\section{References}

1. Bradbury, A. R. M.; Sidhu, S.; Dübel, S.; McCafferty, J., Beyond natural antibodies: the power of in vitro display technologies. Nature Biotechnology 2011, 29 (3), 245-254.

2. Carter, P. J.; Lazar, G. A., Next generation antibody drugs: pursuit of the 'high-hanging fruit'. Nat Rev Drug Discov 2018, 17 (3), 197-223.

3. Boder, E. T.; Jiang, W., Engineering antibodies for cancer therapy. Annu Rev Chem Biomol Eng 2011, 2, 53-75.

4. Chiu, M. L.; Gilliland, G. L., Engineering antibody therapeutics. Curr Opin Struct Biol 2016, 38, 16373.

5. Chiu, M. L.; Goulet, D. R.; Teplyakov, A.; Gilliland, G. L., Antibody Structure and Function: The Basis for Engineering Therapeutics. Antibodies (Basel) 2019, 8 (4).

6. Kennedy, P. J.; Oliveira, C.; Granja, P. L.; Sarmento, B., Antibodies and associates: Partners in targeted drug delivery. Pharmacol Ther 2017, 177, 129-145.

7. Kennedy, P. J.; Oliveira, C.; Granja, P. L.; Sarmento, B., Monoclonal antibodies: technologies for early discovery and engineering. Critical Reviews in Biotechnology 2018, 38 (3), 394-408.

8. Alfaleh, M. A.; Alsaab, H. O.; Mahmoud, A. B.; Alkayyal, A. A.; Jones, M. L.; Mahler, S. M.; Hashem, A. M., Phage Display Derived Monoclonal Antibodies: From Bench to Bedside. Front Immunol 2020, 11, 1986.

9. Almagro, J. C.; Pedraza-Escalona, M.; Arrieta, H. I.; Perez-Tapia, S. M., Phage Display Libraries for Antibody Therapeutic Discovery and Development. Antibodies (Basel) 2019, 8 (3).

10. Bowley, D. R.; Labrijn, A. F.; Zwick, M. B.; Burton, D. R., Antigen selection from an HIV-1 immune antibody library displayed on yeast yields many novel antibodies compared to selection from the same library displayed on phage. Protein Engineering, Design and Selection 2007, 20 (2), 81-90.

11. Boder, E. T.; Raeeszadeh-Sarmazdeh, M.; Price, J. V., Engineering antibodies by yeast display. Archives of Biochemistry and Biophysics 2012, 526 (2), 99-106.

12. Cherf, G. M.; Cochran, J. R., Applications of Yeast Surface Display for Protein Engineering. In Yeast Surface Display: Methods, Protocols, and Applications, Liu, B., Ed. Springer New York: New York, NY, 2015; pp 155-175.

13. Ubah, O.; Palliyil, S., Monoclonal Antibodies and Antibody Like Fragments Derived from Immunised Phage Display Libraries. Adv Exp Med Biol 2017, 1053, 99-117.

14. Tong, Y.; Zhong, S.; Shan, Z.; Yao, W.; Tian, H., A novel human anti-BAFF neutralizing monoclonal antibody derived from in vitro immunization. Biomedicine \& Pharmacotherapy 2019, 119, 109430.

15. McConnell, A. D.; Do, M.; Neben, T. Y.; Spasojevic, V.; MacLaren, J.; Chen, A. P.; Altobell, L.; Macomber, J. L.; Berkebile, A. D.; Horlick, R. A.; Bowers, P. M.; King, D. J., High Affinity Humanized Antibodies without Making Hybridomas; Immunization Paired with Mammalian Cell Display and In Vitro Somatic Hypermutation. Plos One 2012, 7 (11).

16. Ahn, H. J.; Kim, Y.-S.; Lee, C.-H.; Cho, E.-W.; Yoo, H.-S.; Kim, S.-H.; Ko, J.-H.; Kim, S. J., Generation of antibodies recognizing an aberrant glycoform of human tissue inhibitor of metalloproteinase-1 (TIMP-1) using decoy immunization and phage display. Journal of Biotechnology 2011, 151 (2), 225230.

17. Miersch, S.; Sidhu, S. S., Synthetic antibodies: concepts, potential and practical considerations. Methods 2012, 57 (4), 486-98.

18. Adams, J. J.; Sidhu, S. S., Synthetic antibody technologies. Curr Opin Struct Biol 2014, 24, 1-9.

19. Adams, J. J.; Nelson, B.; Sidhu, S. S., Recombinant genetic libraries and human monoclonal antibodies. Methods Mol Biol 2014, 1060, 149-70.

20. Yang, H. Y.; Kang, K. J.; Chung, J. E.; Shim, H., Construction of a large synthetic human scFv library with six diversified CDRs and high functional diversity. Mol Cells 2009, 27 (2), 225-35.

21. Chan, C. E.; Lim, A. P.; MacAry, P. A.; Hanson, B. J., The role of phage display in therapeutic antibody discovery. Int Immunol 2014, 26 (12), 649-57.

22. Prassler, J.; Thiel, S.; Pracht, C.; Polzer, A.; Peters, S.; Bauer, M.; Nörenberg, S.; Stark, Y.; Kölln, J.; Popp, A.; Urlinger, S.; Enzelberger, M., HuCAL PLATINUM, a Synthetic Fab Library Optimized for Sequence Diversity and Superior Performance in Mammalian Expression Systems. Journal of Molecular Biology 2011, 413 (1), 261-278. 
23. Persson, H.; Ye, W.; Wernimont, A.; Adams, J. J.; Koide, A.; Koide, S.; Lam, R.; Sidhu, S. S., CDRH3 Diversity Is Not Required for Antigen Recognition by Synthetic Antibodies. Journal of Molecular Biology 2013, 425 (4), 803-811.

24. Fellouse, F. A.; Barthelemy, P. A.; Kelley, R. F.; Sidhu, S. S., Tyrosine Plays a Dominant Functional Role in the Paratope of a Synthetic Antibody Derived from a Four Amino Acid Code. Journal of Molecular Biology 2006, 357 (1), 100-114.

25. Fellouse, F. A.; Esaki, K.; Birtalan, S.; Raptis, D.; Cancasci, V. J.; Koide, A.; Jhurani, P.; Vasser, M.; Wiesmann, C.; Kossiakoff, A. A.; Koide, S.; Sidhu, S. S., High-throughput Generation of Synthetic Antibodies from Highly Functional Minimalist Phage-displayed Libraries. Journal of Molecular Biology 2007, 373 (4), 924-940.

26. Fellouse, F. A.; Wiesmann, C.; Sidhu, S. S., Synthetic antibodies from a four-amino-acid code: A dominant role for tyrosine in antigen recognition. Proceedings of the National Academy of Sciences of the United States of America 2004, 101 (34), 12467-12472.

27. Mahon, C. M.; Lambert, M. A.; Glanville, J.; Wade, J. M.; Fennell, B. J.; Krebs, M. R.; Armellino, D.; Yang, S.; Liu, X.; O'Sullivan, C. M.; Autin, B.; Oficjalska, K.; Bloom, L.; Paulsen, J.; Gill, D.; Damelin, M.; Cunningham, O.; Finlay, W. J. J., Comprehensive Interrogation of a Minimalist Synthetic CDR-H3 Library and Its Ability to Generate Antibodies with Therapeutic Potential. Journal of Molecular Biology 2013, 425 (10), 1712-1730.

28. Fellouse, F. A.; Li, B.; Compaan, D. M.; Peden, A. A.; Hymowitz, S. G.; Sidhu, S. S., Molecular Recognition by a Binary Code. Journal of Molecular Biology 2005, 348 (5), 1153-1162.

29. Huang, Y.; Wiedmann, M. M.; Suga, H., RNA Display Methods for the Discovery of Bioactive Macrocycles. Chemical Reviews 2019, 119 (17), 10360-10391.

30. Heinis, C.; Winter, G., Encoded libraries of chemically modified peptides. Current Opinion in Chemical Biology 2015, 26, 89-98.

31. Tjhung, K. F.; Kitov, P. I.; Ng, S.; Kitova, E. N.; Deng, L.; Klassen, J. S.; Derda, R., Silent Encoding of Chemical Post-Translational Modifications in Phage-Displayed Libraries. Journal of the American Chemical Society 2016, 138 (1), 32-35.

32. Rezhdo, A.; Islam, M.; Huang, M.; Van Deventer, J. A., Future prospects for noncanonical amino acids in biological therapeutics. Current opinion in biotechnology 2019, 60, 168-178.

33. Krall, N.; da Cruz, F. P.; Boutureira, O.; Bernardes, G. J., Site-selective protein-modification chemistry for basic biology and drug development. Nat Chem 2016, 8 (2), 103-13.

34. Wu, P.; Shui, W. Q.; Carlson, B. L.; Hu, N.; Rabuka, D.; Lee, J.; Bertozzi, C. R., Site-specific chemical modification of recombinant proteins produced in mammalian cells by using the genetically encoded aldehyde tag. Proceedings of the National Academy of Sciences of the United States of America 2009, 106 (9), 3000-3005.

35. ARUNIKA, E.; Lena, S.; Jihea, Y.; Nicholas J., B.; Raja, M.; Atul, B.; Frank, W.; Ratmir, D., Genetically Encoded Fragment-Based Discovery (GE-FBD) from Phage-Displayed Macrocyclic Libraries with Genetically-Encoded Unnatural Pharmacophores. 2020.

36. Navaratna, T.; Atangcho, L.; Mahajan, M.; Subramanian, V.; Case, M.; Min, A.; Tresnak, D.; Thurber, G. M., Directed Evolution Using Stabilized Bacterial Peptide Display. Journal of the American Chemical Society 2020, 142 (4), 1882-1894.

37. Taylor, R. D.; Rey-Carrizo, M.; Passioura, T.; Suga, H., Identification of nonstandard macrocyclic peptide ligands through display screening. Drug Discovery Today: Technologies 2017, 26, 17-23.

38. Hayashi, A.; Haruna, K.-i.; Sato, H.; Ito, K.; Makino, C.; Ito, T.; Sakamoto, K., Incorporation of Halogenated Amino Acids into Antibody Fragments at Multiple Specific Sites Enhances Antigen Binding. ChemBioChem n/a (n/a).

39. Agarwal, P.; Bertozzi, C. R., Site-Specific Antibody-Drug Conjugates: The Nexus of Bioorthogonal Chemistry, Protein Engineering, and Drug Development. Bioconjugate Chemistry 2015, 26 (2), 176 192.

40. Abdollahpour-Alitappeh, M.; Lotfinia, M.; Gharibi, T.; Mardaneh, J.; Farhadihosseinabadi, B.; Larki, P.; Faghfourian, B.; Sepehr, K. S.; Abbaszadeh-Goudarzi, K.; Abbaszadeh-Goudarzi, G.; Johari, B.; Zali, M. R.; Bagheri, N., Antibody-drug conjugates (ADCs) for cancer therapy: Strategies, challenges, and successes. J Cell Physiol 2019, 234 (5), 5628-5642.

41. Hasan, M.; Alam, S.; Poddar, S. K., Antibody-Drug Conjugates: A Review on the Epitome of Targeted Anti- Cancer Therapy. Curr Clin Pharmacol 2018, 13 (4), 236-251. 
42. Beck, A.; Goetsch, L.; Dumontet, C.; Corvaïa, N., Strategies and challenges for the next generation of antibody-drug conjugates. Nature Reviews Drug Discovery 2017, 16 (5), 315-337.

43. Senter, P. D., Potent antibody drug conjugates for cancer therapy. Curr Opin Chem Biol 2009, 13 (3), 235-44.

44. Sievers, E. L.; Senter, P. D., Antibody-drug conjugates in cancer therapy. Annu Rev Med 2013, 64, 15-29.

45. Zhao, P.; Zhang, Y.; Li, W.; Jeanty, C.; Xiang, G.; Dong, Y., Recent advances of antibody drug conjugates for clinical applications. Acta Pharmaceutica Sinica B 2020, 10 (9), 1589-1600.

46. Tian, F.; Lu, Y.; Manibusan, A.; Sellers, A.; Tran, H.; Sun, Y.; Phuong, T.; Barnett, R.; Hehli, B.; Song, F.; DeGuzman, M. J.; Ensari, S.; Pinkstaff, J. K.; Sullivan, L. M.; Biroc, S. L.; Cho, H.; Schultz, P. G.; DiJoseph, J.; Dougher, M.; Ma, D.; Dushin, R.; Leal, M.; Tchistiakova, L.; Feyfant, E.; Gerber, H.-P.; Sapra, P., A general approach to site-specific antibody drug conjugates. Proceedings of the National Academy of Sciences 2014, 111 (5), 1766.

47. Ohri, R.; Bhakta, S.; Fourie-O'Donohue, A.; Dela Cruz-Chuh, J.; Tsai, S. P.; Cook, R.; Wei, B.; Ng, C.; Wong, A. W.; Bos, A. B.; Farahi, F.; Bhakta, J.; Pillow, T. H.; Raab, H.; Vandlen, R.; Polakis, P.; Liu, Y.; Erickson, H.; Junutula, J. R.; Kozak, K. R., High-Throughput Cysteine Scanning To Identify Stable Antibody Conjugation Sites for Maleimide- and Disulfide-Based Linkers. Bioconjug Chem 2018, 29 (2), 473-485.

48. Yamazoe, S.; Hogan, J. M.; West, S. M.; Deng, X. A.; Kotapati, S.; Shao, X.; Holder, P.; Lamba, V.; Huber, M.; Qiang, C.; Gangwar, S.; Rao, C.; Dollinger, G.; Rajpal, A.; Strop, P., High-Throughput Platform to Identify Antibody Conjugation Sites from Antibody-Drug Conjugate Libraries. Bioconjugate Chemistry 2020, 31 (4), 1199-1208.

49. Junutula, J. R.; Bhakta, S.; Raab, H.; Ervin, K. E.; Eigenbrot, C.; Vandlen, R.; Scheller, R. H.; Lowman, H. B., Rapid identification of reactive cysteine residues for site-specific labeling of antibody-Fabs. $J$ Immunol Methods 2008, 332 (1-2), 41-52.

50. Bai, C.; Reid, E. E.; Wilhelm, A.; Shizuka, M.; Maloney, E. K.; Laleau, R.; Harvey, L.; Archer, K. E.; Vitharana, D.; Adams, S.; Kovtun, Y.; Miller, M. L.; Chari, R.; Keating, T. A.; Yoder, N. C., Site-Specific Conjugation of the Indolinobenzodiazepine DGN549 to Antibodies Affords Antibody-Drug Conjugates with an Improved Therapeutic Index as Compared with Lysine Conjugation. Bioconjugate Chemistry 2020, 31 (1), 93-103.

51. Coumans, R. G. E.; Ariaans, G. J. A.; Spijker, H. J.; Renart Verkerk, P.; Beusker, P. H.; Kokke, B. P. A.; Schouten, J.; Blomenröhr, M.; van der Lee, M. M. C.; Groothuis, P. G.; Ubink, R.; Dokter, W. H. A.; Timmers, C. M., A Platform for the Generation of Site-Specific Antibody-Drug Conjugates That Allows for Selective Reduction of Engineered Cysteines. Bioconjugate Chemistry 2020, 31 (9), 21362146.

52. Elledge, S. K.; Tran, H. L.; Christian, A. H.; Steri, V.; Hann, B.; Toste, F. D.; Chang, C. J.; Wells, J. A., Systematic identification of engineered methionines and oxaziridines for efficient, stable, and sitespecific antibody bioconjugation. Proc Natl Acad Sci U S A 2020, 117 (11), 5733-5740.

53. Van Deventer, J. A.; Yuet, K. P.; Yoo, T. H.; Tirrell, D. A., Cell surface display yields evolvable, clickable antibody fragments. Chembiochem 2014, 15 (12), 1777-81.

54. Liu, C. C.; Mack, A. V.; Tsao, M.-L.; Mills, J. H.; Lee, H. S.; Choe, H.; Farzan, M.; Schultz, P. G.; Smider, V. V., Protein evolution with an expanded genetic code. Proceedings of the National Academy of Sciences 2008, 105 (46), 17688-17693.

55. Liu, C. C.; Choe, H.; Farzan, M.; Smider, V. V.; Schultz, P. G., Mutagenesis and Evolution of Sulfated Antibodies Using an Expanded Genetic Code. Biochemistry 2009, 48 (37), 8891-8898.

56. Liu, C. C.; Mack, A. V.; Brustad, E. M.; Mills, J. H.; Groff, D.; Smider, V. V.; Schultz, P. G., Evolution of Proteins with Genetically Encoded "Chemical Warheads". Journal of the American Chemical Society 2009, 131 (28), 9616-9617.

57. Li, X.; Hitomi, J.; Liu, C. C., Characterization of a Sulfated Anti-HIV Antibody Using an Expanded Genetic Code. Biochemistry 2018, 57 (20), 2903-2907.

58. Rader, C.; Sinha, S. C.; Popkov, M.; Lerner, R. A.; Barbas, C. F., Chemically programmed monoclonal antibodies for cancer therapy: Adaptor immunotherapy based on a covalent antibody catalyst. Proceedings of the National Academy of Sciences 2003, 100 (9), 5396-5400.

59. Doppalapudi, V. R.; Huang, J.; Liu, D.; Jin, P.; Liu, B.; Li, L.; Desharnais, J.; Hagen, C.; Levin, N. J.; Shields, M. J.; Parish, M.; Murphy, R. E.; Del Rosario, J.; Oates, B. D.; Lai, J.-Y.; Matin, M. J.; Ainekulu, 
Z.; Bhat, A.; Bradshaw, C. W.; Woodnutt, G.; Lerner, R. A.; Lappe, R. W., Chemical generation of bispecific antibodies. Proceedings of the National Academy of Sciences 2010, 107 (52), 22611-22616.

60. Jespers, L.; Bonnert, T. P.; Winter, G., Selection of optical biosensors from chemisynthetic antibody libraries. Protein Eng Des Sel 2004, 17 (10), 709-13.

61. Cheng, A. C.; Doherty, E. M.; Johnstone, S.; DiMauro, E. F.; Dao, J.; Luthra, A.; Ye, J.; Tang, J.; Nixey, T.; Min, X.; Tagari, P.; Miranda, L. P.; Wang, Z., Structure-guided Discovery of Dual-recognition Chemibodies. Scientific Reports 2018, 8 (1), 7570.

62. Van Deventer, J. A.; Le, D. N.; Zhao, J.; Kehoe, H. P.; Kelly, R. L., A platform for constructing, evaluating, and screening bioconjugates on the yeast surface. Protein Eng Des Sel 2016, 29 (11), 485-494.

63. Stieglitz, J. T.; Kehoe, H. P.; Lei, M.; Van Deventer, J. A., A Robust and Quantitative Reporter System To Evaluate Noncanonical Amino Acid Incorporation in Yeast. ACS synthetic biology 2018, 7 (9), 2256-2269.

64. Potts, K. A.; Stieglitz, J. T.; Lei, M.; Van Deventer, J. A., Reporter system architecture affects measurements of noncanonical amino acid incorporation efficiency and fidelity. Molecular Systems Design \& Engineering 2020, 5 (2), 573-588.

65. Kelly, R. L.; Le, D.; Zhao, J.; Wittrup, K. D., Reduction of Nonspecificity Motifs in Synthetic Antibody Libraries. J Mol Biol 2018, 430 (1), 119-130.

66. Nam, D. H.; Rodriguez, C.; Remacle, A. G.; Strongin, A. Y.; Ge, X., Active-site MMP-selective antibody inhibitors discovered from convex paratope synthetic libraries. Proceedings of the National Academy of Sciences 2016, 113 (52), 14970.

67. Van Deventer, J. A.; Kelly, R. L.; Rajan, S.; Wittrup, K. D.; Sidhu, S. S., A switchable yeast display/secretion system. Protein Engineering, Design and Selection 2015, 28 (10), 317-325.

68. Dai, Z.; Lai, J. R., Isolation of Synthetic Antibodies Against BCL-2-Associated X Protein (BAX). In BCL2 Family Proteins: Methods and Protocols, Gavathiotis, E., Ed. Springer New York: New York, NY, 2019; pp 351-357.

69. Woldring, D. R.; Holec, P. V.; Zhou, H.; Hackel, B. J., High-Throughput Ligand Discovery Reveals a Sitewise Gradient of Diversity in Broadly Evolved Hydrophilic Fibronectin Domains. PLoS One 2015, 10 (9), e0138956.

70. Kabat, E. A.; Wu, T. T., Identical $V$ region amino acid sequences and segments of sequences in antibodies of different specificities. Relative contributions of $\mathrm{VH}$ and $\mathrm{VL}$ genes, minigenes, and complementarity-determining regions to binding of antibody-combining sites. The Journal of Immunology 1991, 147 (5), 1709-1719.

71. Eigenbrot, C.; Randal, M.; Presta, L.; Carter, P.; Kossiakoff, A. A., X-ray Structures of the Antigenbinding Domains from Three Variants of Humanized anti-p185HER2 Antibody 4D5 and Comparison with Molecular Modeling. Journal of Molecular Biology 1993, 229 (4), 969-995.

72. Van Deventer, J. A.; Wittrup, K. D., Yeast surface display for antibody isolation: library construction, library screening, and affinity maturation. Methods Mol Biol 2014, 1131, 151-81.

73. Chao, G.; Lau, W. L.; Hackel, B. J.; Sazinsky, S. L.; Lippow, S. M.; Wittrup, K. D., Isolating and engineering human antibodies using yeast surface display. Nature Protocols 2006, 1 (2), 755-768.

74. Quartararo, A. J.; Gates, Z. P.; Somsen, B. A.; Hartrampf, N.; Ye, X.; Shimada, A.; Kajihara, Y.; Ottmann, C.; Pentelute, B. L., Ultra-large chemical libraries for the discovery of high-affinity peptide binders. Nat Commun 2020, 11 (1), 3183.

75. Hackel, B. J.; Wittrup, K. D., The full amino acid repertoire is superior to serine/tyrosine for selection of high affinity immunoglobulin $\mathrm{G}$ binders from the fibronectin scaffold. Protein Engineering, Design and Selection 2010, 23 (4), 211-219.

76. Kruziki, Max A.; Bhatnagar, S.; Woldring, Daniel R.; Duong, Vandon T.; Hackel, Benjamin J., A 45Amino-Acid Scaffold Mined from the PDB for High-Affinity Ligand Engineering. Chemistry \& Biology 2015, 22 (7), 946-956.

77. Arranz-Gibert, P.; Vanderschuren, K.; Isaacs, F. J., Next-generation genetic code expansion. Current Opinion in Chemical Biology 2018, 46, 203-211.

78. Des Soye, B. J.; Patel, J. R.; Isaacs, F. J.; Jewett, M. C., Repurposing the translation apparatus for synthetic biology. Current Opinion in Chemical Biology 2015, 28, 83-90.

79. Vargas-Rodriguez, O.; Sevostyanova, A.; Söll, D.; Crnković, A., Upgrading aminoacyl-tRNA synthetases for genetic code expansion. Current Opinion in Chemical Biology 2018, 46, 115-122. 
80. Bryson, D. I.; Fan, C.; Guo, L.-T.; Miller, C.; Söll, D.; Liu, D. R., Continuous directed evolution of aminoacyl-tRNA synthetases. Nature Chemical Biology 2017, 13 (12), 1253-1260.

81. Monk, J. W.; Leonard, S. P.; Brown, C. W.; Hammerling, M. J.; Mortensen, C.; Gutierrez, A. E.; Shin, N. Y.; Watkins, E.; Mishler, D. M.; Barrick, J. E., Rapid and Inexpensive Evaluation of Nonstandard Amino Acid Incorporation in Escherichia coli. ACS synthetic biology 2017, 6 (1), 45-54.

82. Kang, M.; Lu, Y.; Chen, S.; Tian, F., Harnessing the power of an expanded genetic code toward nextgeneration biopharmaceuticals. Current Opinion in Chemical Biology 2018, 46, 123-129.

83. Ryu, Y.; Schultz, P. G., Efficient incorporation of unnatural amino acids into proteins in Escherichia coli. Nature Methods 2006, 3 (4), 263-265.

84. Dunbar, J.; Krawczyk, K.; Leem, J.; Baker, T.; Fuchs, A.; Georges, G.; Shi, J.; Deane, C. M., SAbDab: the structural antibody database. Nucleic Acids Research 2014, 42 (D1), D1140-D1146.

85. Yu, C. M.; Peng, H. P.; Chen, I. C.; Lee, Y. C.; Chen, J. B.; Tsai, K. C.; Chen, C. T.; Chang, J. Y.; Yang, E. W.; Hsu, P. C.; Jian, J. W.; Hsu, H. J.; Chang, H. J.; Hsu, W. L.; Huang, K. F.; Ma, A. C.; Yang, A. S., Rationalization and design of the complementarity determining region sequences in an antibody-antigen recognition interface. PLoS One 2012, 7 (3), e33340.

86. Wilkinson, I. C.; Hall, C. J.; Veverka, V.; Shi, J. Y.; Muskett, F. W.; Stephens, P. E.; Taylor, R. J.; Henry, A. J.; Carr, M. D., High Resolution NMR-based Model for the Structure of a scFv-IL-1 beta Complex POTENTIAL FOR NMR AS A KEY TOOL IN THERAPEUTIC ANTIBODY DESIGN AND DEVELOPMENT. J Biol Chem 2009, 284 (46), 31928-31935.

87. Ganesan, R.; Eigenbrot, C.; Wu, Y.; Liang, W. C.; Shia, S.; Lipari, M. T.; Kirchhofer, D., Unraveling the allosteric mechanism of serine protease inhibition by an antibody. Structure 2009, 17 (12), 16141624.

88. Kudo, S.; Caaveiro, J. M.; Nagatoishi, S.; Miyafusa, T.; Matsuura, T.; Sudou, Y.; Tsumoto, K., Disruption of cell adhesion by an antibody targeting the cell-adhesive intermediate (X-dimer) of human P-cadherin. Sci Rep 2017, 7, 39518.

89. Wu, N.; Deiters, A.; Cropp, T. A.; King, D.; Schultz, P. G., A genetically encoded photocaged amino acid. J Am Chem Soc 2004, 126 (44), 14306-7.

90. Lemke, E. A.; Summerer, D.; Geierstanger, B. H.; Brittain, S. M.; Schultz, P. G., Control of protein phosphorylation with a genetically encoded photocaged amino acid. Nat Chem Biol 2007, 3 (12), 76972.

91. Lang, K.; Chin, J. W., Bioorthogonal reactions for labeling proteins. ACS Chem Biol 2014, 9 (1), 16 20.

92. McKay, C. S.; Finn, M. G., Click chemistry in complex mixtures: bioorthogonal bioconjugation. Chem Biol 2014, 21 (9), 1075-101.

93. Devaraj, N. K., The Future of Bioorthogonal Chemistry. ACS Cent Sci 2018, 4 (8), 952-959.

94. Jung, S.; Kwon, I., Expansion of bioorthogonal chemistries towards site-specific polymer-protein conjugation. Polym Chem-Uk 2016, 7 (28), 4584-4598.

95. Agard, N. J.; Baskin, J. M.; Prescher, J. A.; Lo, A.; Bertozzi, C. R., A comparative study of bioorthogonal reactions with azides. ACS Chem Biol 2006, 1 (10), 644-8.

96. Agard, N. J.; Prescher, J. A.; Bertozzi, C. R., A strain-promoted [3 + 2] azide-alkyne cycloaddition for covalent modification of biomolecules in living systems. J Am Chem Soc 2004, 126 (46), 15046-7.

97. Jewett, J. C.; Bertozzi, C. R., Cu-free click cycloaddition reactions in chemical biology. Chem Soc Rev 2010, 39 (4), 1272-9.

98. Hong, V.; Steinmetz, N. F.; Manchester, M.; Finn, M. G., Labeling live cells by copper-catalyzed alkyne--azide click chemistry. Bioconjug Chem 2010, 21 (10), 1912-6.

99. Li, L.; Zhang, Z. Y., Development and Applications of the Copper-Catalyzed Azide-Alkyne Cycloaddition (CuAAC) as a Bioorthogonal Reaction. Molecules 2016, 21 (10).

100. Farrer, N. J.; Griffith, D. M., Exploiting azide-alkyne click chemistry in the synthesis, tracking and targeting of platinum anticancer complexes. Curr Opin Chem Biol 2020, 55, 59-68.

101. Kislukhin, A. A.; Hong, V. P.; Breitenkamp, K. E.; Finn, M. G., Relative performance of alkynes in copper-catalyzed azide-alkyne cycloaddition. Bioconjug Chem 2013, 24 (4), 684-9.

102. Zimmerman, E. S.; Heibeck, T. H.; Gill, A.; Li, X. F.; Murray, C. J.; Madlansacay, M. R.; Tran, C.; Uter, N. T.; Yin, G.; Rivers, P. J.; Yam, A. Y.; Wang, W. D.; Steiner, A. R.; Bajad, S. U.; Penta, K.; Yang, W. J.; Hallam, T. J.; Thanos, C. D.; Sato, A. K., Production of Site-Specific Antibody-Drug Conjugates Using Optimized Non-Natural Amino Acids in a Cell-Free Expression System. Bioconjugate Chemistry 2014, 25 (2), 351-361. 
103. Coin, I., Application of non-canonical crosslinking amino acids to study protein-protein interactions in live cells. Current Opinion in Chemical Biology 2018, 46, 156-163.

104. Mishra, P. K.; Yoo, C. M.; Hong, E.; Rhee, H. W., Photo-crosslinking: An Emerging Chemical Tool for Investigating Molecular Networks in Live Cells. Chembiochem 2020, 21 (7), 924-932.

105. Murale, D. P.; Hong, S. C.; Haque, M. M.; Lee, J.-S., Photo-affinity labeling (PAL) in chemical proteomics: a handy tool to investigate protein-protein interactions (PPIs). Proteome Science 2017, $15(1), 14$.

106. Ray-Saha, S.; Huber, T.; Sakmar, T. P., Antibody Epitopes on G Protein-Coupled Receptors Mapped with Genetically Encoded Photoactivatable Cross-Linkers. Biochemistry 2014, 53 (8), 1302-1310.

107. Berg, M.; Michalowski, A.; Palzer, S.; Rupp, S.; Sohn, K., An in vivo photo-cross-linking approach reveals a homodimerization domain of Aha1 in S. cerevisiae. PLoS One 2014, 9 (3), e89436.

108. Futran, A. S.; Kyin, S.; Shvartsman, S. Y.; Link, A. J., Mapping the binding interface of ERK and transcriptional repressor Capicua using photocrosslinking. Proceedings of the National Academy of Sciences 2015, 112 (28), 8590-8595.

109. Taupitz, K. F.; Dörner, W.; Mootz, H. D., Covalent Capturing of Transient SUMO-SIM Interactions Using Unnatural Amino Acid Mutagenesis and Photocrosslinking. Chemistry - A European Journal 2017, 23 (25), 5978-5982.

110. Choi, C. P.; Moon, A. S.; Back, P. S.; Jami-Alahmadi, Y.; Vashisht, A. A.; Wohlschlegel, J. A.; Bradley, P. J., A photoactivatable crosslinking system reveals protein interactions in the Toxoplasma gondii inner membrane complex. PLoS Biol 2019, 17 (10), e3000475.

111. Shin, G.; Lim, S. I., Site-specific proximity ligation provides molecular insights into biologically relevant interfaces of protein-protein interaction. Biochemical and Biophysical Research Communications 2020.

112. Ackerman, M.; Levary, D.; Tobon, G.; Hackel, B.; Orcutt, K. D.; Wittrup, K. D., Highly avid magnetic bead capture: An efficient selection method for de novo protein engineering utilizing yeast surface display. Biotechnology Progress 2009, 25 (3), 774-783.

113. Stern, L. A.; Csizmar, C. M.; Woldring, D. R.; Wagner, C. R.; Hackel, B. J., Titratable Avidity Reduction Enhances Affinity Discrimination in Mammalian Cellular Selections of Yeast-Displayed Ligands. ACS Combinatorial Science 2017, 19 (5), 315-323.

114. Lim, S.; Glasgow, J. E.; Filsinger Interrante, M.; Storm, E. M.; Cochran, J. R., Dual display of proteins on the yeast cell surface simplifies quantification of binding interactions and enzymatic bioconjugation reactions. Biotechnology Journal 2017, 12 (5), 1600696.

115. Niquille, D. L.; Hansen, D. A.; Mori, T.; Fercher, D.; Kries, H.; Hilvert, D., Nonribosomal biosynthesis of backbone-modified peptides. Nature Chemistry 2018, 10 (3), 282-287.

116. Link, A. J.; Tirrell, D. A., Cell Surface Labeling of Escherichia coli via Copper(I)-Catalyzed [3+2] Cycloaddition. Journal of the American Chemical Society 2003, 125 (37), 11164-11165.

117. Tanrikulu, I. C.; Schmitt, E.; Mechulam, Y.; Goddard, W. A., 3rd; Tirrell, D. A., Discovery of Escherichia coli methionyl-tRNA synthetase mutants for efficient labeling of proteins with azidonorleucine in vivo. Proc Natl Acad Sci U S A 2009, 106 (36), 15285-90.

118. Golinski, A. W.; Holec, P. V.; Mischler, K. M.; Hackel, B. J., Biophysical Characterization Platform Informs Protein Scaffold Evolvability. ACS Combinatorial Science 2019, 21 (4), 323-335.

119. Silverman, A. P.; Levin, A. M.; Lahti, J. L.; Cochran, J. R., Engineered Cystine-Knot Peptides that Bind avß3 Integrin with Antibody-Like Affinities. Journal of Molecular Biology 2009, 385 (4), 1064-1075.

120. Miller, E. A.; Sung, K.-J.; Kongsuphol, P.; Baniya, S.; Aw-Yong, H. Q.; Tay, V.; Tan, Y.; Kabir, F. M.; Pang-Yeo, K.; Kaspriskie, I. G.; Sikes, H. D., Beyond Epitope Binning: Directed in Vitro Selection of Complementary Pairs of Binding Proteins. ACS Combinatorial Science 2020, 22 (1), 49-60.

121. Plückthun, A., Designed Ankyrin Repeat Proteins (DARPins): Binding Proteins for Research, Diagnostics, and Therapy. Annual Review of Pharmacology and Toxicology 2015, 55 (1), 489-511.

122. McMahon, C.; Baier, A. S.; Pascolutti, R.; Wegrecki, M.; Zheng, S.; Ong, J. X.; Erlandson, S. C.; Hilger, D.; Rasmussen, S. G. F.; Ring, A. M.; Manglik, A.; Kruse, A. C., Yeast surface display platform for rapid discovery of conformationally selective nanobodies. Nature Structural \& Molecular Biology 2018, 25 (3), 289-296.

123. Hoyt, E. A.; Cal, P. M. S. D.; Oliveira, B. L.; Bernardes, G. J. L., Contemporary approaches to siteselective protein modification. Nature Reviews Chemistry 2019, 3 (3), 147-171.

124. Chen, S.; Bogyo, M., A phage display approach to identify highly selective covalent binders. bioRxiv 2019, 791533. 
125. Tharp, J. M.; Hampton, J. T.; Reed, C. A.; Ehnbom, A.; Chen, P.-H. C.; Morse, J. S.; Kurra, Y.; Pérez, L. M.; Xu, S.; Liu, W. R., An amber obligate active site-directed ligand evolution technique for phage display. Nature Communications 2020, 11 (1), 1392.

126. Wang, X. S.; Chen, P.-H. C.; Hampton, J. T.; Tharp, J. M.; Reed, C. A.; Das, S. K.; Wang, D.-S.; Hayatshahi, H. S.; Shen, Y.; Liu, J.; Liu, W. R., A Genetically Encoded, Phage-Displayed CyclicPeptide Library. Angewandte Chemie International Edition 2019, 58 (44), 15904-15909.

127. Passioura, T.; Liu, W.; Dunkelmann, D.; Higuchi, T.; Suga, H., Display Selection of Exotic Macrocyclic Peptides Expressed under a Radically Reprogrammed 23 Amino Acid Genetic Code. Journal of the American Chemical Society 2018, 140 (37), 11551-11555.

128. Owens, A. E.; lannuzzelli, J. A.; Gu, Y.; Fasan, R., MOrPH-PhD: An Integrated Phage Display Platform for the Discovery of Functional Genetically Encoded Peptide Macrocycles. ACS Central Science 2020, 6 (3), 368-381.

129. Bacon, K.; Blain, A.; Burroughs, M.; McArthrur, N.; Rao, B. M.; Menegatti, S., Isolation of Chemically Cyclized Peptide Binders Using Yeast Surface Display. ACS Combinatorial Science 2020, 22 (10), 519-532.

130. Ng, S.; Lin, E.; Kitov, P. I.; Tjhung, K. F.; Gerlits, O. O.; Deng, L.; Kasper, B.; Sood, A.; Paschal, B. M.; Zhang, P.; Ling, C.-C.; Klassen, J. S.; Noren, C. J.; Mahal, L. K.; Woods, R. J.; Coates, L.; Derda, R., Genetically Encoded Fragment-Based Discovery of Glycopeptide Ligands for CarbohydrateBinding Proteins. Journal of the American Chemical Society 2015, 137 (16), 5248-5251.

131. Deyle, K.; Kong, X.-D.; Heinis, C., Phage Selection of Cyclic Peptides for Application in Research and Drug Development. Accounts of Chemical Research 2017, 50 (8), 1866-1874.

132. Zhu, E. F.; Gai, S. A.; Opel, C. F.; Kwan, B. H.; Surana, R.; Mihm, M. C.; Kauke, M. J.; Moynihan, K. D.; Angelini, A.; Williams, R. T.; Stephan, M. T.; Kim, J. S.; Yaffe, M. B.; Irvine, D. J.; Weiner, L. M.; Dranoff, G.; Wittrup, K. D., Synergistic innate and adaptive immune response to combination immunotherapy with anti-tumor antigen antibodies and extended serum half-life IL-2. Cancer Cell 2015, 27 (4), 489-501.

133. Hong, V.; Presolski, S. I.; Ma, C.; Finn, M. G., Analysis and optimization of copper-catalyzed azidealkyne cycloaddition for bioconjugation. Angew Chem Int Ed Engl 2009, 48 (52), 9879-83. 


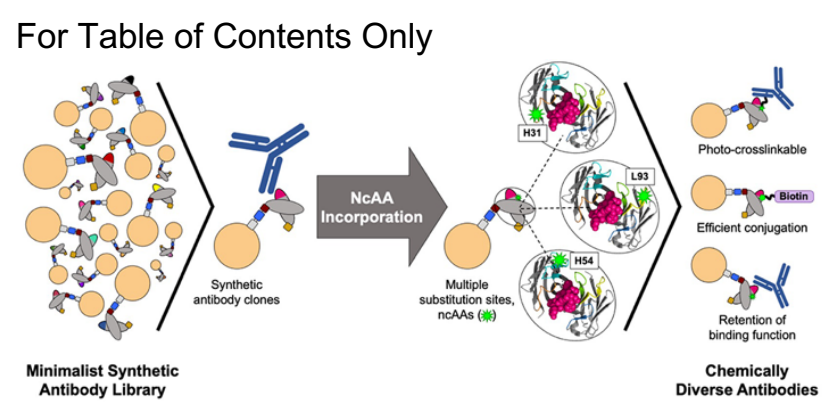

